\title{
ENTIRE SOLUTIONS FOR CRITICAL $p$-FRACTIONAL HARDY SCHRÖDINGER KIRCHHOFF EQUATIONS
}

\author{
Paolo Piersanti and Patrizia Pucci
}

\begin{abstract}
Existence theorems of nonnegative entire solutions of stationary critical $p$-fractional Hardy Schrödinger Kirchhoff equations are presented in this paper. The equations we treat deal with Hardy terms and critical nonlinearities and the main theorems extend several recent results on the topic. The paper contains also some open problems.
\end{abstract}

2010 Mathematics Subject Classification: Primary: 35R11, 35J60; Secondary: 35J20, 35B09.

Key words: Stationary Kirchhoff problems, nonlocal $p$-Laplacian operators, Hardy coefficients, critical exponents.

\section{Introduction}

Nonlinear problems involving fractional diffusions appear in several areas of applied mathematics, as described by Caffarelli in $[\mathbf{8}, \mathbf{9}]$ and by Vázquez in $[\mathbf{4 2}, \mathbf{4 3}]$. Indeed, many recent papers are devoted to problems involving fractional and nonlocal operators, and concerning models in optimization, finance, continuum mechanics, phase transition phenomena, population dynamics, and game theory. Several contributions have also been given for nonlinear fractional Schrödinger equations, fractional porous medium equations, and general nonlinear problems of any type. For a recent survey on some up-to-date developments we refer to the recent survey $[\mathbf{3 9}]$.

In particular, the paper deals with stationary fractional Kirchhoff $p$ Laplacian equations, involving critical nonlinearities, a topic of great appeal after the publication of the paper [22] due to Fiscella and Valdinoci. We refer e.g. to $[\mathbf{2}, \mathbf{1 0}, \mathbf{1 6}, \mathbf{2 9}, \mathbf{3 0}, \mathbf{3 2}, \mathbf{3 6}, \mathbf{3 7}]$ and the references therein for details. But the equations treated here contain also Hardy terms, which make the analysis more delicate and quite interesting. For related problems we just mention $[\mathbf{5}, \mathbf{1 0}, \mathbf{1 1}, \mathbf{2 1}]$ and the references cited in there. 
Since we are interested in nonnegative entire solutions for applications in geometry and physics, the first equation we treat is of critical $p$-fractional Hardy Schrödinger Kirchhoff type, that is

$$
\begin{aligned}
M\left(\|u\|^{p}\right)\left[(-\Delta)_{p}^{s} u\right. & \left.+V(x)|u|^{p-2} u\right]-\gamma \frac{|u|^{p-2} u}{|x|^{p s}} \\
= & \lambda f(x, u)+g(x, u)+K(x)\left(u^{+}\right)^{p_{s}^{*}-1} \text { in } \mathbb{R}^{N},
\end{aligned}
$$

where $\gamma$ and $\lambda$ are real parameters, $0<s<1<p<\infty$, sp $<N$, and $u^{+}=\max \{u, 0\}$. The operator $(-\Delta)_{p}^{s}$ is the fractional $p$-Laplacian, which for every function $\varphi \in C_{0}^{\infty}\left(\mathbb{R}^{N}\right)$ may be defined, up to normalization factors, as

$$
(-\Delta)_{p}^{s} \varphi(x)=2 \lim _{\varepsilon \rightarrow 0^{+}} \int_{\mathbb{R}^{N} \backslash B_{\varepsilon}(x)} \frac{|\varphi(x)-\varphi(y)|^{p-2}[\varphi(x)-\varphi(y)]}{|x-y|^{N+p s}} d y
$$

for all $x \in \mathbb{R}^{N}$, where $B_{\varepsilon}(x)=\left\{y \in \mathbb{R}^{N}:|x-y|<\varepsilon\right\}$. The exponent $p_{s}^{*}=N p /(N-p s)$ is critical in the sense of Sobolev, while the nonlinear terms $f$ and $g$ are subcritical. For all $\varphi \in C_{0}^{\infty}\left(\mathbb{R}^{N}\right)$

$$
\begin{gathered}
\|\varphi\|^{p}=[\varphi]_{s, p}^{p}+\|\varphi\|_{p, V}^{p}, \quad\|\varphi\|_{p, V}^{p}=\int_{\mathbb{R}^{N}} V(x)|u(x)|^{p} d x, \\
{[\varphi]_{s, p}^{p}=\iint_{\mathbb{R}^{2 N}} \frac{|\varphi(x)-\varphi(y)|^{p}}{|x-y|^{N+p s}} d x d y .}
\end{gathered}
$$

Throughout the paper the weights $K$ and $V$ satisfy

(K) $K \geq 0$ a.e. in $\mathbb{R}^{N}$ and $K \in L^{\infty}\left(\mathbb{R}^{N}\right)$,

$(V) V \in C\left(\mathbb{R}^{N}\right)$ and $V(x) \geq V_{0}>0$ for all $x \in \mathbb{R}^{N}$, where $V_{0}$ is a positive constant,

while the main Kirchhoff function $M$ verifies the condition

$(\mathcal{M}) M: \mathbb{R}_{0}^{+} \rightarrow \mathbb{R}_{0}^{+}$is a nonnegative continuous function and there exists $\theta \in[1, N /(N-p s))$ such that $t M(t) \leq \theta \mathscr{M}(t)$ for any $t \in \mathbb{R}_{0}^{+}$, where $\mathscr{M}(t)=\int_{0}^{t} M(\tau) d \tau$.

Note that $\|\cdot\|_{p, V}$ is a uniformly convex norm on the weighted Lebesgue Banach space $L^{p}\left(\mathbb{R}^{N}, V\right)$ by $(V)$.

Problem (1.1) is fairly delicate due to the intrinsic lack of compactness, which arise from the Hardy term and the nonlinearity with critical exponent $p_{s}^{*}$. For this reason we assume that (1.1) is non-degenerate, that is

$$
\inf _{t \in \mathbb{R}_{0}^{+}} M(t)=a>0 \text {. }
$$


Stationary non-degenerate Kirchhoff problems have been extensively studied in the last decades, but usually under the request that $M$ is nondecreasing in $\mathbb{R}_{0}^{+}$, as in $[\mathbf{2 2}, \mathbf{3 3}, \mathbf{3 5}]$ and the reference therein. As in $[\mathbf{2}, \mathbf{1 0}, \mathbf{3 6}]$ the monotonicity assumption is replaced by $(\mathcal{M})$. In particular, as shown in $[\mathbf{2}, \mathbf{3 6}]$, the Kirchhoff function $M(t)=(1+t)^{k}+$ $(1+t)^{-1}, k \in(0,1)$, verifies both $(\mathcal{M})$ and $(1.3)$, but is not monotone. Indeed, $\inf _{t \in \mathbb{R}_{0}^{+}} M(t)=a>0$, with $a=k^{-k /(k+1)}(1+k)<M(0)=2$, and $(\mathcal{M})$ is satisfied, with $\theta=k+1$ and $k$ so small that $k<s p /(N-s p)$.

The main framework for (1.1) is the space $E$, defined as the completion of $C_{0}^{\infty}\left(\mathbb{R}^{N}\right)$ with respect to the norm $\|\cdot\|$, introduced in (1.2). Denote by $D^{s, p}\left(\mathbb{R}^{N}\right)$ the $p$-fractional Beppo-Levi space, that is the completion of $C_{0}^{\infty}\left(\mathbb{R}^{N}\right)$ with respect to Gagliardo semi-norm $[\cdot]_{s, p}$. Theorems 1 and 2 of [31] give

$$
\begin{gathered}
\|u\|_{p_{s}^{*}}^{p} \leq C_{N, p} \frac{s(1-s)}{(N-p s)^{p-1}}[u]_{s, p}^{p}, \quad\|u\|_{H}^{p} \leq C_{N, p} \frac{s(1-s)}{(N-p s)^{p}}[u]_{s, p}^{p}, \\
\|u\|_{H}^{p}=\int_{\mathbb{R}^{N}} \frac{|u(x)|^{p}}{|x|^{p s}} d x
\end{gathered}
$$

for all $u \in D^{s, p}\left(\mathbb{R}^{N}\right)$, where $C_{N, p}$ is a positive constant depending only on $N$ and $p$. Thus, the fractional Sobolev embedding $D^{s, p}\left(\mathbb{R}^{N}\right) \hookrightarrow$ $L^{p_{s}^{*}}\left(\mathbb{R}^{N}\right)$ and the fractional Hardy embedding $D^{s, p}\left(\mathbb{R}^{N}\right) \hookrightarrow L^{p}\left(\mathbb{R}^{N},|x|^{-p s}\right)$ are continuous, but not compact. It is also evident that $E \hookrightarrow D^{s, p}\left(\mathbb{R}^{N}\right)$. Let us introduce the best fractional critical Sobolev and Hardy constants $S=S(N, p, s)$ and $H=H(N, p, s)$ given by

$$
S=\inf _{\substack{u \in D^{s, p}\left(\mathbb{R}^{N}\right) \\ u \neq 0}} \frac{[u]_{s, p}^{p}}{\|u\|_{p_{s}^{*}}^{p}}, \quad H=\inf _{\substack{u \in D^{s, p}\left(\mathbb{R}^{N}\right) \\ u \neq 0}} \frac{[u]_{s, p}^{p}}{\|u\|_{H}^{p}} .
$$

Of course, the numbers $S$ and $H$ are strictly positive. We refer to Theorem 1.1 of [23] for the sharp Hardy constant $H$. Throughout the paper we require the following structural assumptions on $f$ and $g$.

$(\mathcal{F}) f: \mathbb{R}^{N} \times \mathbb{R} \rightarrow \mathbb{R}$ is a Carathéodory function and there exists an exponent $q \in\left(\theta p, p_{s}^{*}\right)$ such that either

$\left(f_{1}\right) f(x, t)=w(x)\left(t^{+}\right)^{q-1}$ for a.a. $x \in \mathbb{R}^{N}$ and all $t \in \mathbb{R}$, where $w>0$ a.e. in $\mathbb{R}^{N}$ and $w \in L^{\wp}\left(\mathbb{R}^{N}\right)$, with $\wp=p_{s}^{*} /\left(p_{s}^{*}-q\right)$, or

$\left(f_{2}\right) f$ verifies both assumptions

(a) there exists a positive function $w$ of class $L^{\infty}\left(\mathbb{R}^{N}\right)$ such that $w(x)=o(1)$ as $|x| \rightarrow \infty$ and $|f(x, t)| \leq w(x)|t|^{q-1}$ for a.a. $x \in \mathbb{R}^{N}$ and all $t \in \mathbb{R}$, 
(b) for a.a. $x \in \mathbb{R}^{N}$ it results $0<q F(x, t) \leq t f(x, t)$ for all $t \in \mathbb{R}^{+}$and $0 \leq q F(x, t) \leq t f(x, t)$ for all $t \in \mathbb{R}_{0}^{-}$, where $F(x, t)=\int_{0}^{t} f(x, \tau) d \tau$.

(G) $g: \mathbb{R}^{N} \times \mathbb{R} \rightarrow \mathbb{R}$ is a Carathéodory function and there exist exponents $r$ and $\mu$ in $\left(\theta p, p_{s}^{*}\right)$ such that for all $\varepsilon>0$ there exists $C_{\varepsilon}>0$ and

$$
|g(x, t)| \leq \theta p \varepsilon|t|^{\theta p-1}+r C_{\varepsilon}|t|^{r-1}
$$

for a.a. $x \in \mathbb{R}^{N}$ and all $t \in \mathbb{R}$, and either

(i) $\theta p<\mu<q$ and $\mu G(x, t) \leq t g(x, t)$ for a.a. $x \in \mathbb{R}^{N}$ and all $t \in \mathbb{R}$, where $G(x, t)=\int_{0}^{t} g(x, \tau) d \tau$, or

(ii) $q \leq \mu<p_{s}^{*}$ and $0 \leq \mu G(x, t) \leq t g(x, t)$ for a.a. $x \in \mathbb{R}^{N}$ and all $t \in \mathbb{R}$.

For examples of subcritical nonlinear terms which satisfy conditions $(\mathcal{F})$ and $(\mathcal{G})$ we refer to $[\mathbf{1 0}]$. The condition, assumed in [36], namely $\inf \left\{G(x, t): x \in \mathbb{R}^{N},|t|=1\right\}>0$, is no longer required here and in [10] thanks to the possible presence of the nontrivial nonlinearity $f$.

For the next main existence result for (1.1), because of the possible presence of $g$, we assume also

$(\mathcal{V})$ There exists $R>0$ such that for any $c>0$

$$
\lim _{|y| \rightarrow \infty} \operatorname{meas}\left(\left\{x \in B_{R}(y): V(x) \leq c\right\}\right)=0 .
$$

Condition $(\mathcal{V})$ is weaker than the property $V(x) \rightarrow \infty$ as $|x| \rightarrow \infty$ usually required in Schrödinger problems. Assumption $(\mathcal{V})$ was originally introduced by Bartsch and Wang in [6] to overcome the lack of compactness in problems defined in the entire space $\mathbb{R}^{N}$.

In harmony with $[\mathbf{1 0}]$, we define $\kappa=\kappa(q, \mu, M)$ by

$$
\kappa=\frac{a(\tau-\theta p)}{\theta(\tau-p)}, \quad \tau=\min \{q, \mu\} .
$$

Clearly $\kappa \in(0, a]$, being $\theta \geq 1$ and $p \leq \theta p<\tau$ by assumptions $(\mathcal{F})$ and $(\mathcal{G})$. There are cases, besides the obvious one $M \equiv a$, in which $\kappa=a$, that is $\theta=1$ in $(\mathcal{M})$, as shown in Section 2 of [10].

Thanks to the variational nature of (1.1), under the above structural assumptions, (weak) solutions of (1.1) are exactly the critical points of the underlying functional $J_{\gamma, \lambda}$, which satisfies the geometry of the mountain pass lemma. The solutions constructed for problem (1.1) are given in terms of critical points $u_{\gamma, \lambda}$ of $J_{\gamma, \lambda}$ determined at special mountain pass levels. These solutions are briefly called mountain pass solutions. 
Theorem 1.1. Suppose that (1.1) is non-degenerate, i.e., that (1.3) holds, and that $(\mathcal{M}),(V),(\mathcal{V}),(\mathcal{F})$, and $(\mathcal{G})$ are satisfied. Then for every $\gamma \in(-\infty, \kappa H)$ problem (1.1) admits a nontrivial mountain pass solution $u_{\gamma, \lambda}$ for any $\lambda>0$, whenever $\|K\|_{\infty}=0$, and $u_{\gamma, \lambda}$ satisfies the asymptotic behavior

$$
\lim _{\lambda \rightarrow \infty}\left\|u_{\gamma, \lambda}\right\|=0
$$

While if $\|K\|_{\infty}>0$, then there exists $\lambda^{*}=\lambda^{*}(\gamma)>0$ such that for any $\lambda \geq \lambda^{*}$ problem (1.1) admits a nontrivial mountain pass solution $u_{\gamma, \lambda}$ which satisfies again (1.6).

Moreover, if $g \equiv 0$, then the assertion above continues to hold assuming only condition $(V)$ on the potential $V$.

Finally, if $f, g: \mathbb{R}^{N} \times \mathbb{R} \rightarrow \mathbb{R}$ are Carathéodory functions, with the property that for a.a. $x \in \mathbb{R}^{N}$

$$
\begin{gathered}
f(x, t)=g(x, t)=0 \text { for all } t \in \mathbb{R}_{0}^{-}, \\
f(x, t)>0 \text { and } g(x, t) \geq 0 \text { for all } t \in \mathbb{R}^{+},
\end{gathered}
$$

and if $h$ is a nonnegative perturbation term of class $L^{\nu^{\prime}}\left(\mathbb{R}^{N}\right)$, where $\nu^{\prime}$ is the conjugate exponent of some fixed $\nu \in\left[p, p_{s}^{*}\right]$, then the nonhomogeneous equation associated with (1.1), that is

$$
\begin{aligned}
M\left(\|u\|^{p}\right) & {\left[(-\Delta)_{p}^{s} u+V(x)|u|^{p-2} u\right]-\gamma \frac{|u|^{p-2} u}{|x|^{p s}} } \\
& =\lambda f(x, u)+g(x, u)+K(x)\left(u^{+}\right)^{p_{s}^{*}-1}+h(x) \text { in } \mathbb{R}^{N},
\end{aligned}
$$

admits only nonnegative solutions in $\mathbb{R}^{N}$, provided that $\lambda \geq 0$ and $\gamma<a H$.

Therefore, every nontrivial mountain pass solution $u_{\gamma, \lambda}$ constructed in the first part of Theorem 1.1 is nonnegative in $\mathbb{R}^{N}$, being $\gamma<\kappa H \leq a H$, whenever also (1.7) holds. Theorem 1.1 extends in several directions, for example, Theorem 1.1 of [13], Theorem 1.1 of $[\mathbf{1 6}]$, Theorem 1.1 of $[\mathbf{1 9}]$, Theorem 1.3 of [21], Theorems 1.1 and 1.2 of [25], Theorem 1.2 parts (2) and $(3)$ of $[\mathbf{3 0}]$, Theorem 1.1 of $[\mathbf{3 6}]$, and $[\mathbf{1 2}, \mathbf{1 8}, \mathbf{2 4}, \mathbf{2 9}, \mathbf{4 0}, \mathbf{4 5}]$.

The last part of Theorem 1.1, that is when $g \equiv 0$ in (1.1), takes somehow inspiration from the paper [41] and covers also the interesting case in which $V$ is a positive constant. See also Theorem 1.1 of $[\mathbf{1 0}]$. Theorem 1.1 completes the picture given in Theorem 1.1 of [20].

As in $[\mathbf{1 0}]$, we can study equation (1.1), requiring only $(V)$ on the potential $V$, but including the term $g$, provided that $K, V, f$, and $g$ are radial functions in $x$. 
Theorem 1.2. Let $N \geq 2$ and let (1.3) hold. Assume that $(\mathcal{M}),(V)$, $(\mathcal{F})$, and $(\mathcal{G})$ are satisfied and that $K, V, f$, and $g$ are radial functions in $x$. Then for every $\gamma \in(-\infty, \kappa H)$ problem (1.1) admits a nontrivial radial mountain pass solution $u_{\gamma, \lambda}$ for any $\lambda>0$ and $u_{\gamma, \lambda}$ satisfies the asymptotic behavior (1.6), whenever $\|K\|_{\infty}=0$. While if $\|K\|_{\infty}>0$, then there exists $\lambda^{*}=\lambda^{*}(\gamma)>0$ such that for any $\lambda \geq \lambda^{*}$ problem (1.1) admits a nontrivial radial mountain pass solution $u_{\gamma, \lambda}$, which satisfies again (1.6).

Theorem 1.2 extends in several directions the existence results obtained in $[\mathbf{1 7}, \mathbf{2 6}, \mathbf{2 7}, \mathbf{4 4}]$ and the references cited therein.

The second problem we consider comes from the equation

$$
\begin{aligned}
& M\left(\|u\|^{p}\right)\left[(-\Delta)_{p}^{s} u+V(x)|u|^{p-2} u\right]-\gamma \frac{|u|^{p-2} u}{|x|^{p s}} \\
& =\lambda w(x)\left(u^{+}\right)^{q-1}+K(x)\left(u^{+}\right)^{p_{s}^{*}-1}+h(x) \text { in } \mathbb{R}^{N},
\end{aligned}
$$

where the parameters satisfy the previous assumptions, and $f(x, t)=$ $w(x)\left(t^{+}\right)^{q-1}$ is as in $(\mathcal{F})-\left(f_{1}\right)$, that is $q \in\left(\theta p, p_{s}^{*}\right)$, the weight $w>0$ a.e. in $\mathbb{R}^{N}$ and of class $L^{\wp}\left(\mathbb{R}^{N}\right)$, with $\wp=p_{s}^{*} /\left(p_{s}^{*}-q\right)$. The function $h$ can be viewed again as a nonnegative perturbation term and $h$ is assumed in the second part of the paper to be nontrivial, nonnegative and of class $L^{\nu^{\prime}}\left(\mathbb{R}^{N}\right)$, where $\nu^{\prime}$ is the conjugate exponent of some fixed $\nu \in\left[p, p_{s}^{*}\right]$.

For problem (1.9) we also assume for simplicity that $K>0$ a.e. in $\mathbb{R}^{N}$ and crucially that $M$ is in standard form, that is there exists $\theta \in[1, N /(N-p s))$ such that

$$
M(t)=a+b \theta t^{\theta-1}, \quad a>0, b \geq 0,
$$

for all $t \in \mathbb{R}_{0}^{+}$. Clearly, condition (1.10) is stronger than the previous assumption $(\mathcal{M})$.

Theorem 1.3. Suppose that $(V)$ and (1.10) hold. Then for all $\gamma$ in $(-\infty, a H)$ and $\lambda>0$ there exists $\delta=\delta\left(\gamma^{+}, \lambda\right)>0$ such that for all nontrivial nonnegative perturbations $h \in L^{\nu^{\prime}}\left(\mathbb{R}^{N}\right)$, with $0<\|h\|_{\nu^{\prime}} \leq \delta$, problem (1.9) admits a nontrivial nonnegative solution $u_{\gamma, \lambda}$, provided that either $\theta=1$ in (1.10), or $\theta>1$ and $\gamma \leq 0$. Furthermore, the solution $u_{\gamma, \lambda}$ satisfies $(1.6)$.

Theorem 1.3 extends Theorem 1.1 of [36], the existence result to obtain the first solution of Theorem 1.3, as well as Theorem 1.4 parts (2) and $(3)$ of $[\mathbf{3 0}]$. 
A very natural appealing open problem is to prove existence of nontrivial solutions for problems (1.1), (1.8), and (1.9) in the degenerate case, that is when $M(0)=0$ and $M(t)>0$ for all $t>0$.

The paper is organized as follows. In Section 2 we prove the existence Theorem 1.1 for the Hardy Schrödinger Kirchhoff problem (1.1) and the asymptotic behavior (1.6). Section 3 deals with the proof of Theorem 1.3. Finally, in Section 4 we extend Theorems 1.1 and 1.3 to settings having wider applications, replacing the fractional $p$-Laplacian operator by a general nonlocal integro-differential operator, generated by a singular kernel $\mathcal{K}$, satisfying the natural assumptions described by Caffarelli, e.g., in $[8]$. See also $[\mathbf{3 9}]$.

\section{The non-degenerate Hardy-Schrödinger-Kirchhoff equation (1.1)}

Here we prove the existence result for problem (1.1) and we recall that throughout this section $(\mathcal{M}),(K),(V)$, and (1.3) hold. First, by Theorem 6.7 and Corollary 7.2 of [15] we have the following embedding result for the uniformly convex Banach space $E$ defined in the introduction. The fact that $E$ is a uniformly convex Banach space can be easily derived following the main arguments of Proposition A.9 of [3], or Lemma 10 of [36], or Lemma A.6 of [37].

Lemma 2.1. If $\nu \in\left[p, p_{s}^{*}\right]$, then the embeddings $E \hookrightarrow W^{s, p}\left(\mathbb{R}^{N}\right) \hookrightarrow$ $L^{\nu}\left(\mathbb{R}^{N}\right)$ are continuous. In particular, there exists a constant $C_{\nu}>0$ such that $\|u\|_{\nu} \leq C_{\nu}\|u\|$ for all $u \in E$.

Let $L^{q}\left(\mathbb{R}^{N}, w\right)$ be the weighted Lebesgue space, endowed with the norm

$$
\|u\|_{q, w}^{q}=\int_{\mathbb{R}^{N}} w(x)|u|^{q} d x .
$$

The Banach space $L^{q}\left(\mathbb{R}^{N}, w\right)=\left(L^{q}\left(\mathbb{R}^{N}, w\right),\|\cdot\|_{q, w}\right)$ is uniformly convex by Proposition A.6 of [3]. Furthermore, by Lemma 2.1 of [10], which combines some ideas of Lemma 2.3 of [3], Lemma 2.2 of [4], and Lemma 2.6 of [38], see also Lemma 2.3 of [37], the embedding $D^{s, p}\left(\mathbb{R}^{N}\right) \hookrightarrow L^{q}\left(\mathbb{R}^{N}, w\right)$ is compact, with

$$
\|u\|_{q, w} \leq C_{w}[u]_{s, p} \quad \text { for all } u \in D^{s, p}\left(\mathbb{R}^{N}\right)
$$

and $C_{w}=S^{-1 / p}\|w\|_{\wp}^{1 / q}>0$. 
We say that $u \in E$ is a (weak) solution of (1.1) if $u$ satisfies the identity

$$
\begin{aligned}
M\left(\|u\|^{p}\right)\langle u, \varphi\rangle-\gamma\langle u, \varphi\rangle_{H}= & \lambda \int_{\mathbb{R}^{N}} f(x, u(x)) \varphi(x) d x \\
& +\int_{\mathbb{R}^{N}} g(x, u(x)) \varphi(x) d x+\left\langle u^{+}, \varphi\right\rangle_{p_{s}^{*}, K}
\end{aligned}
$$

for any $\varphi \in E$, where $\langle u, \varphi\rangle=\langle u, \varphi\rangle_{s, p}+\langle u, \varphi\rangle_{p, V}$,

$$
\begin{aligned}
\langle u, \varphi\rangle_{s, p} & =\iint_{\mathbb{R}^{2 N}} \frac{|u(x)-u(y)|^{p-2}[u(x)-u(y)] \cdot[\varphi(x)-\varphi(y)]}{|x-y|^{N+p s}} d x d y, \\
\langle u, \varphi\rangle_{p, V} & =\int_{\mathbb{R}^{N}} V(x)|u(x)|^{p-2} u(x) \varphi(x) d x, \\
\langle u, \varphi\rangle_{H} & =\int_{\mathbb{R}^{N}} \frac{|u(x)|^{p-2} u(x) \varphi(x)}{|x|^{p s}} d x, \\
\left\langle u^{+}, \varphi\right\rangle_{p_{s}^{*}, K} & =\int_{\mathbb{R}^{N}} K(x)\left(u^{+}(x)\right)^{p_{s}^{*}-1} \varphi(x) d x .
\end{aligned}
$$

Problem (1.1) has a variational structure and $J_{\gamma, \lambda}: E \rightarrow \mathbb{R}$, defined by

$$
\begin{aligned}
J_{\gamma, \lambda}(u) & =\frac{1}{p}\left[\mathscr{M}\left(\|u\|^{p}\right)-\gamma\|u\|_{H}^{p}\right]-H_{\lambda}(u), \\
H_{\lambda}(u) & =\lambda \int_{\mathbb{R}^{N}} F(x, u) d x+\int_{\mathbb{R}^{N}} G(x, u) d x+\frac{1}{p_{s}^{*}}\left\|u^{+}\right\|_{p_{s}^{*}, K}^{p^{*}}, \\
\left\|u^{+}\right\|_{p_{s}^{*}, K}^{p^{*}} & =\int_{\mathbb{R}^{N}} K(x)\left(u^{+}(x)\right)^{p_{s}^{*}} d x,
\end{aligned}
$$

is the underlying functional associated with (1.1). Indeed, $J_{\gamma, \lambda}$ is well defined in $E$, since if $u \in E$, then also $u^{+} \in E$ and $u^{-} \in E$, being

$\left|u^{+}(x)-u^{+}(y)\right| \leq|u(x)-u(y)| \quad$ and $\quad\left|u^{-}(x)-u^{-}(y)\right| \leq|u(x)-u(y)|$

for a.a. $x, y \in \mathbb{R}^{N}$. Essentially, as shown in Lemmas 4.2 of [10], the functional $J_{\gamma, \lambda}$ is of class $C^{1}(E)$.

Condition (1.3) gives that $M(t)>0$ for any $t \in \mathbb{R}_{0}^{+}$and $(\mathcal{M})$ yields that $t \mapsto t^{-\theta} \mathscr{M}(t)$ is nonincreasing in $\mathbb{R}^{+}$. Consequently, for all $t_{0}>0$

$$
t_{0}^{\theta} \mathscr{M}(t) \leq \mathscr{M}\left(t_{0}\right) t^{\theta} \quad \text { for any } t \geq t_{0} .
$$

Now, as in Lemmas 2.2 and 4.3 of [10], we prove that the functional $J_{\gamma, \lambda}$ has the geometric features required to apply the mountain pass theorem of Ambrosetti and Rabinowitz of [1]. To this aim, let us note that the 
assumption $(\mathcal{G})$ implies

$$
|G(x, t)| \leq \varepsilon|t|^{\theta p}+C_{\varepsilon}|t|^{r} \quad \text { for a.a. } x \in \mathbb{R}^{N} \text { and all } t \in \mathbb{R} .
$$

Lemma 2.2. Fix $\gamma \in(-\infty, a H)$ and $\lambda>0$. Then there exists a radial function $e \in C_{0}^{\infty}\left(\mathbb{R}^{N}\right)$, with $e \geq 0$ in $\mathbb{R}^{N}$, $\|e\| \geq 2$, and $J_{\gamma, \lambda}(e)<0$, which depends only on $\gamma^{-}$, when $K>0$ a.e. in $\mathbb{R}^{N}$. Furthermore, there exist $\alpha=\alpha\left(\gamma^{+}, \lambda\right)>0$ and $\rho=\rho\left(\gamma^{+}, \lambda\right) \in(0,1]$ such that $J_{\gamma, \lambda}(u) \geq \alpha$ for all $u \in E$, with $\|u\|=\rho$.

Proof: Fix $\gamma \in(-\infty, a H)$ and $\lambda>0$. Take a radial function $v$ of class $C_{0}^{\infty}\left(\mathbb{R}^{N}\right)$, with $v \geq 0$ in $\mathbb{R}^{N}$ and $\|v\|=1$. As shown in the proof of Lemma 4.3 of [10], for a.a. $x \in \mathbb{R}^{N}$ the functions $t \mapsto t^{-q} F(x, t v(x))$ and $t \mapsto t^{-\mu} G(x, t v(x))$ are nondecreasing in $\mathbb{R}^{+}$by $(\mathcal{F})$ and by $(\mathcal{G})$, in both cases (i) and (ii). Moreover, $\int_{\mathbb{R}^{N}} F(x, v) d x>0$ by $(\mathcal{F})$ and the fact that $v \in C_{0}^{\infty}\left(\mathbb{R}^{N}\right), v \geq 0$ in $\mathbb{R}^{N}$, and $\|v\|=1$. Hence, as $t \rightarrow \infty$

$$
\int_{\mathbb{R}^{N}} F(x, t v) d x \geq t^{q} \int_{\mathbb{R}^{N}} F(x, v) d x \rightarrow \infty .
$$

While $(\mathcal{G})$, in both cases (i) and (ii), implies that

$$
\int_{\mathbb{R}^{N}} G(x, t v) d x \geq t^{\mu} \int_{\mathbb{R}^{N}} G(x, v) d x \quad \text { for all } t \geq 1 .
$$

Consequently, by (2.3) for all $t \geq 1$

$$
\begin{aligned}
J_{\gamma, \lambda}(t v) \leq & \frac{\mathscr{M}(1)}{p} t^{\theta p}+\frac{\gamma^{-}}{p}\|v\|_{H}^{p} t^{p}-\lambda \int_{\mathbb{R}^{N}} F(x, t v) d x \\
& -\int_{\mathbb{R}^{N}} G(x, t v) d x-\frac{\left\|v^{+}\right\|_{p_{s}^{*}, K}^{p^{*}}}{p_{s}^{*}} t^{p_{s}^{*}} \\
\leq & \frac{\mathscr{M}(1)}{p} t^{\theta p}+\frac{\gamma^{-}}{p}\|v\|_{H}^{p} t^{p}-\frac{\left\|v^{+}\right\|_{p_{s}^{*}, K}^{p^{*}}}{p_{s}^{*}} t^{p_{s}^{*}} \\
& - \begin{cases}\left(\lambda t^{q} \int_{\mathbb{R}^{N}} F(x, v) d x+t^{\mu} \int_{\mathbb{R}^{N}} G(x, v) d x\right) & \operatorname{under}(\mathcal{G})-(\mathrm{i}) \\
\lambda t^{q} \int_{\mathbb{R}^{N}} F(x, v) d x & \text { under }(\mathcal{G})-(\mathrm{ii})\end{cases}
\end{aligned}
$$

as $t \rightarrow \infty$, since $p \leq \theta p<\min \{q, \mu\} \leq \max \{q, \mu\}<p_{s}^{*}$ by $(\mathcal{F}),(\mathcal{G})$, and (2.5), even if $K=0$ a.e. in $\mathbb{R}^{N}$. Choosing $e=\tau_{0} v$, with $\tau_{0}>0$ large enough, we get at once that $e$ is regular, radial, $e \geq 0$ in $\mathbb{R}^{N},\|e\| \geq 2$, and $J_{\gamma, \lambda}(e)<0$. Clearly $e$ depends on $\gamma^{-}$. Furthermore, $e$ can be taken independent of $\lambda$, whenever $K>0$ a.e. in $\mathbb{R}^{N}$, otherwise $e$ could depend also on $\lambda$, as claimed. 
By $(K),(V),(1.3),(1.4),(2.4)$, and the fact that $E \hookrightarrow D^{s, p}\left(\mathbb{R}^{N}\right)$, there exists a positive constant $K_{w}$ by $(\mathcal{F})$, such that for all $u \in E$, with $\|u\| \leq 1$,

$$
\begin{aligned}
J_{\gamma, \lambda}(u) & \geq \frac{a}{p}\|u\|^{p}-\frac{\gamma}{p}\|u\|_{H}^{p}-\lambda K_{w}\|u\|^{q}-\varepsilon\|u\|_{\theta p}^{\theta p}-C_{\varepsilon}\|u\|_{r}^{r}-\frac{\left\|u^{+}\right\|_{p_{s}^{*}, K}^{p_{s}^{*}}}{p_{s}^{*}} \\
& \geq\left(\frac{a}{p}-\frac{\gamma^{+}}{p H}-\varepsilon C_{\theta p}^{\theta p}\right)\|u\|^{p}-\lambda K_{w}\|u\|^{q}-C_{\varepsilon} C_{r}^{r}\|u\|^{r}-S_{K}\|u\|^{p_{s}^{*}} \\
& \geq \beta\|u\|^{p}-\lambda K_{w}\|u\|^{q}-C_{\varepsilon} C_{r}^{r}\|u\|^{r}-S_{K}\|u\|^{p_{s}^{*}},
\end{aligned}
$$

since $\|u\|^{\theta p} \leq\|u\|^{p}$, being $p \leq \theta p$ and $\|u\| \leq 1$. Here

$$
S_{K}=\frac{\|K\|_{\infty}}{p_{s}^{*} S_{s}^{p_{s}^{*} / p}}, \quad \varepsilon=\frac{a H-\gamma^{+}}{2 p H C_{\theta p}^{\theta p}}>0, \quad \beta=\frac{1}{2 p}\left(a-\frac{\gamma^{+}}{H}\right)>0,
$$

since $\gamma<a H$. The function

$$
\eta_{\gamma, \lambda}(t)=\beta t^{p}-\lambda K_{w} t^{q}-C_{\varepsilon} C_{r}^{r} t^{r}-S_{K} t^{p_{s}^{*}}, \quad t \in[0,1],
$$

admits a maximum at some $\rho \in(0,1]$ small enough, that is

$$
\max _{t \in[0,1]} \eta_{\gamma, \lambda}(t)=\eta_{\gamma, \lambda}(\rho)>0,
$$

since $\beta>0$ and $p \leq \theta p<\min \{q, r\} \leq \max \{q, r\}<p_{s}^{*}$ by $(\mathcal{M}),(\mathcal{F})$, and $(\mathcal{G})$. Put $\alpha=\eta_{\gamma, \lambda}(\rho)$. Consequently, $J_{\gamma, \lambda}(u) \geq \alpha>0$ for all $u \in E$, with $\|u\|=\rho$.

From the proof of Lemma 2.2 it is apparent that if $e$ is the nontrivial nonnegative radial function determined for some $\gamma \in(-\infty, a H)$ and $\lambda_{0}>0$, then $e$ is such that $J_{\gamma, \lambda}(e)<0$ for all $\lambda \geq \lambda_{0}$ and $\|e\| \geq 2>\rho=$ $\rho\left(\gamma^{+}, \lambda\right)$, being $\rho \in(0,1]$.

We recall in passing that, if $X$ is a real Banach space, a $C^{1}(X)$ functional $J$ satisfies the Palais-Smale condition at level $c \in \mathbb{R}$ if any PalaisSmale sequence $\left(u_{n}\right)_{n}$ at level $c$, that is any sequence $\left(u_{n}\right)_{n}$, with the property that

$$
J\left(u_{n}\right) \rightarrow c \text { and } \quad J^{\prime}\left(u_{n}\right) \rightarrow 0 \quad \text { in } X^{\prime} \text { as } n \rightarrow \infty,
$$

admits a strongly convergent subsequence in $X$.

Fix $\gamma \in(-\infty, a H), \lambda>0$ and put

$$
\begin{aligned}
c_{\gamma, \lambda} & =\inf _{\xi \in \Gamma} \max _{t \in[0,1]} J_{\gamma, \lambda}(\xi(t)), \\
\Gamma & =\{\xi \in C([0,1], E): \xi(0)=0, \xi(1)=e\} .
\end{aligned}
$$

Obviously, $c_{\gamma, \lambda}>0$ thanks to Lemma 2.2 . 
We are going to prove that $J_{\gamma, \lambda}$ satisfies the Palais-Smale condition at level $c_{\gamma, \lambda}$ in $E$. To this aim, we show an asymptotic property of the levels $c_{\gamma, \lambda}$. This crucial idea is strongly related to Lemma 2.3 of [10] (see also Lemma 4.3 of [21] and Lemma 6 of [22] for a somehow similar fractional non-degenerate Kirchhoff Dirichlet problem in bounded regular domains). The next lemma indeed is useful to obtain (1.6) and, most importantly, to defeat the lack of compactness due to the presence of a Hardy term and a critical nonlinearity. In order to get the Palais-Smale condition at level $c_{\gamma, \lambda}$ in $E$, the presence of $g$ forces to also assume $(\mathcal{V})$ on the potential $V$, as we shall see in the proof of the main Lemma 2.4.

Lemma 2.3. For all $\gamma \in(-\infty, a H)$ it results

$$
\lim _{\lambda \rightarrow \infty} c_{\gamma, \lambda}=0 .
$$

Proof: Fix $\gamma \in(-\infty, a H)$ and $\lambda_{0}>0$. Let $e \in C_{0}^{\infty}\left(\mathbb{R}^{N}\right)$ be the nontrivial nonnegative regular radial function obtained in Lemma 2.2, depending on $\gamma^{-}$and possibly on $\lambda_{0}$. Hence the functional $J_{\gamma, \lambda}$ satisfies the mountain pass geometry at 0 and $e$ for all $\lambda \geq \lambda_{0}$. In particular, there exists $t_{\gamma, \lambda}>0$ such that $J_{\gamma, \lambda}\left(t_{\gamma, \lambda} e\right)=\max _{t \geq 0} J_{\gamma, \lambda}(t e)$. Therefore, $\left\langle J_{\gamma, \lambda}^{\prime}\left(t_{\gamma, \lambda} e\right), e\right\rangle_{E^{\prime}, E}=0$, that is

$$
\begin{aligned}
0= & \left\langle J_{\gamma, \lambda}^{\prime}\left(t_{\gamma, \lambda} e\right), e\right\rangle_{E^{\prime}, E}=M\left(t_{\gamma, \lambda}^{p}\|e\|^{p}\right)\left\langle t_{\gamma, \lambda} e, e\right\rangle-\gamma t_{\gamma, \lambda}^{p-1}\|e\|_{H}^{p} \\
& -t_{\gamma, \lambda}^{p_{s}^{*}-1}\|e\|_{p_{s}^{*}, K}^{p_{*}^{*}}-\lambda \int_{\mathbb{R}^{N}} f\left(x, t_{\gamma, \lambda} e\right) e d x-\int_{\mathbb{R}^{N}} g\left(x, t_{\gamma, \lambda} e\right) e d x
\end{aligned}
$$

for all $\lambda \geq \lambda_{0}$.

We claim that $\left\{t_{\gamma, \lambda}\right\}_{\lambda \geq \lambda_{0}}$ is bounded in $\mathbb{R}$. To this aim let us define $\Lambda=\left\{\lambda \geq \lambda_{0}: t_{\gamma, \lambda} \geq 1\right\}$. Then for all $\lambda \in \Lambda$, by $(\mathcal{M}),(\mathcal{F}),(\mathcal{G}),(1.4)$, (2.3), (2.5), and (2.6)

$$
\begin{aligned}
t_{\gamma, \lambda}^{\theta p-1} & \left(\theta \mathscr{M}(1)+\frac{\gamma^{-}}{H}\right)\|e\|^{\theta p} \\
& \geq t_{\gamma, \lambda}^{p-1}\left(M\left(t_{\gamma, \lambda}^{p}\|e\|^{p}\right)\|e\|^{p}-\gamma\|e\|_{H}^{p}\right) \\
& \geq \lambda t_{\gamma, \lambda}^{q-1} q \int_{\mathbb{R}^{N}} F(x, e) d x+t_{\gamma, \lambda}^{\mu-1} \mu \int_{\mathbb{R}^{N}} G(x, e) d x+t_{\gamma, \lambda}^{p_{s}^{*}-1}\|e\|_{p_{s}^{*}, K}^{p_{s}^{*}} \\
& \geq t_{\gamma, \lambda}^{q-1} \lambda_{0} q \int_{\mathbb{R}^{N}} F(x, e) d x+ \begin{cases}t_{\gamma, \lambda}^{\mu-1} \mu \int_{\mathbb{R}^{N}} G(x, e) d x & \text { under }(\mathcal{G}) \text {-(i) } \\
0 & \text { under }(\mathcal{G}) \text {-(ii) }\end{cases}
\end{aligned}
$$

since $\int_{\mathbb{R}^{N}} F(x, e) d x>0$ and $\|e\| \geq 2$. This implies at once the boundedness of $\left\{t_{\gamma, \lambda}\right\}_{\lambda \in \Lambda}$, since $\theta p<q$ by $(\mathcal{F})$ and $q<\mu$ in the case $(\mathcal{G})$-(i). In particular, this shows that $\left\{t_{\gamma, \lambda}\right\}_{\lambda \geq \lambda_{0}}$ is bounded and proves the claim. 
Fix a sequence $\left(\lambda_{k}\right)_{k} \subset\left[\lambda_{0}, \infty\right)$ such that $\lambda_{k} \rightarrow \infty$ as $k \rightarrow \infty$. Obviously $\left(t_{\gamma, \lambda_{k}}\right)_{k}$ is bounded in $\mathbb{R}$. Hence, there exists a subsequence of $\left(\lambda_{k}\right)_{k}$, still relabeled $\left(\lambda_{k}\right)_{k}$, and a number $\ell \geq 0$ such that $t_{\gamma, \lambda_{k}} \rightarrow \ell$ as $k \rightarrow \infty$. Assume by contradiction that $\ell>0$. Then, by $(\mathcal{F}),(\mathcal{G})$, and the Lebesgue dominated convergence theorem, we obtain

$$
\begin{aligned}
& \lim _{k \rightarrow \infty} \int_{\mathbb{R}^{N}} f\left(x, t_{\gamma, \lambda_{k}} e\right) e d x=\int_{\mathbb{R}^{N}} f(x, \ell e) e d x>0, \\
& \lim _{k \rightarrow \infty} \int_{\mathbb{R}^{N}} g\left(x, t_{\gamma, \lambda_{k}} e\right) e d x=\int_{\mathbb{R}^{N}} g(x, \ell e) e d x,
\end{aligned}
$$

since $e \geq 0$ in $\mathbb{R}^{N}$ and $\|e\| \geq 2$. Hence, (2.8) and the argument above gives

$$
\begin{array}{r}
\ell^{\theta p-1}\left(\theta \mathscr{M}(1)+\frac{\gamma^{-}}{H}\right)\|e\|^{\theta p} \geq \lim _{k \rightarrow \infty}\left(\lambda_{k} \int_{\mathbb{R}^{N}} f\left(x, t_{\gamma, \lambda_{k}} e\right) e d x\right) \\
+\int_{\mathbb{R}^{N}} g(x, \ell e) e d x=\infty,
\end{array}
$$

which is the desired contradiction. In conclusion, $\ell=0$, being the sequence $\left(\lambda_{k}\right)_{k}$, with $\lambda_{k} \rightarrow \infty$, arbitrary.

Consider now the path $\xi(t)=t e, t \in[0,1]$, belonging to $\Gamma$. By Lemma $2.2,(\mathcal{F})$, and $(2.6)$

$$
\begin{aligned}
0<c_{\gamma, \lambda} \leq \max _{t \in[0,1]} J_{\gamma, \lambda}(t e) \leq J_{\gamma, \lambda}\left(t_{\gamma, \lambda} e\right) \leq & \frac{1}{p} \mathscr{M}\left(t_{\gamma, \lambda}^{p}\|e\|^{p}\right)+\frac{\gamma^{-}}{p}\|e\|_{H}^{p} t_{\gamma, \lambda}^{p} \\
& +t_{\gamma, \lambda}^{\mu}\left[\int_{\mathbb{R}^{N}} G(x, e) d x\right]^{-} .
\end{aligned}
$$

Moreover, $\mathscr{M}\left(t_{\gamma, \lambda}^{p}\|e\|^{p}\right) \rightarrow 0$ as $\lambda \rightarrow \infty$, by the continuity of $\mathscr{M}$ and the fact that $t_{\gamma, \lambda}=o(1)$ as $\lambda \rightarrow \infty$. This completes the proof of the lemma, since $e$ is independent of $\lambda \geq \lambda_{0}$.

Now, following the key idea of the proof of Lemmas 2.4 and 4.5 in [10], we prove the validity of the Palais-Smale condition for $J_{\gamma, \lambda}$ at level $c_{\gamma, \lambda}$ in $E$. The crucial argument also appears in the proof of Lemma 4.5 in [21], given for Dirichlet problems in bounded domains, when $M \equiv 1$ and $p=2$. Let us recall that $\kappa$ is the constant given in (1.5).

Lemma 2.4. Assume also $(\mathcal{V})$ and let $\gamma \in(-\infty, \kappa H)$ be fixed. If $\|K\|_{\infty}=0$, then $J_{\gamma, \lambda}$ satisfies the Palais-Smale condition at level $c_{\gamma, \lambda}$ for all $\lambda>0$. While if $\|K\|_{\infty}>0$, then there exists $\lambda^{*}=\lambda^{*}(\gamma)>0$ such that $J_{\gamma, \lambda}$ satisfies the Palais-Smale condition at level $c_{\gamma, \lambda}$ for any $\lambda \geq \lambda^{*}$. 
Proof: Fix $\gamma<\kappa H, \lambda>0$, and let $\left(u_{n}\right)_{n} \subset E$ be a Palais-Smale sequence of $J_{\gamma, \lambda}$ at level $c_{\gamma, \lambda}$. Assume first that $\theta p<\mu<q$, so that $\tau=\min \{q, \mu\}=\mu$ in (1.5). Thus, by $(\mathcal{M}),(\mathcal{F}),(\mathcal{G})-(\mathrm{i})$, and (1.3) we get

$$
\begin{aligned}
J_{\gamma, \lambda}\left(u_{n}\right) & -\frac{1}{\mu}\left\langle J_{\gamma, \lambda}^{\prime}\left(u_{n}\right), u_{n}\right\rangle_{E^{\prime}, E} \geq\left(\frac{1}{\theta p}-\frac{1}{\mu}\right) M\left(\left\|u_{n}\right\|^{p}\right)\left\|u_{n}\right\|^{p} \\
& -\gamma\left(\frac{1}{p}-\frac{1}{\mu}\right)\left\|u_{n}\right\|_{H}^{p}-\lambda \int_{\mathbb{R}^{N}} F\left(x, u_{n}\right) d x+\frac{\lambda}{\mu} \int_{\mathbb{R}^{N}} f\left(x, u_{n}\right) u_{n} d x \\
& -\int_{\mathbb{R}^{N}} G\left(x, u_{n}\right) d x+\frac{1}{\mu} \int_{\mathbb{R}^{N}} g\left(x, u_{n}\right) u_{n} d x+\left(\frac{1}{\mu}-\frac{1}{p_{s}^{*}}\right)\left\|u_{n}^{+}\right\|_{p_{s}^{*}, K}^{p^{*}} \\
\geq & a\left(\frac{1}{\theta p}-\frac{1}{\mu}\right)\left\|u_{n}\right\|^{p}-\frac{\gamma^{+}}{H}\left(\frac{1}{p}-\frac{1}{\mu}\right)\left\|u_{n}\right\|^{p} \\
& -\lambda \int_{\mathbb{R}^{N}} F\left(x, u_{n}\right) d x+\frac{\lambda}{q} \int_{\mathbb{R}^{N}} f\left(x, u_{n}\right) u_{n} d x+\left(\frac{1}{\mu}-\frac{1}{p_{s}^{*}}\right)\left\|u_{n}^{+}\right\|_{p_{s}^{*}, K}^{p_{s}^{*}} \\
\geq & {\left[a\left(\frac{1}{\theta p}-\frac{1}{\mu}\right)-\frac{\gamma^{+}}{H}\left(\frac{1}{p}-\frac{1}{\mu}\right)\right]\left\|u_{n}\right\|^{p}+\left(\frac{1}{\mu}-\frac{1}{p_{s}^{*}}\right)\left\|u_{n}^{+}\right\|_{p_{s}^{*}, K}^{p_{s}^{*}} . }
\end{aligned}
$$

Similarly, if $q \leq \mu<p_{s}^{*}$, so that $\tau=\min \{q, \mu\}=q$ in (1.5), replacing now $(\mathcal{G})$-(i) by $(\mathcal{G})$-(ii), we have

$$
\begin{aligned}
J_{\gamma, \lambda}\left(u_{n}\right) & -\frac{1}{q}\left\langle J_{\gamma, \lambda}^{\prime}\left(u_{n}\right), u_{n}\right\rangle_{E^{\prime}, E} \geq a\left(\frac{1}{\theta p}-\frac{1}{q}\right)\left\|u_{n}\right\|^{p} \\
& -\frac{\gamma^{+}}{H}\left(\frac{1}{p}-\frac{1}{q}\right)\left\|u_{n}\right\|^{p}-\lambda \int_{\mathbb{R}^{N}} F\left(x, u_{n}\right) d x+\frac{\lambda}{q} \int_{\mathbb{R}^{N}} f\left(x, u_{n}\right) u_{n} d x \\
& -\int_{\mathbb{R}^{N}} G\left(x, u_{n}\right) d x+\frac{1}{\mu} \int_{\mathbb{R}^{N}} g\left(x, u_{n}\right) u_{n} d x+\left(\frac{1}{q}-\frac{1}{p_{s}^{*}}\right)\left\|u_{n}^{+}\right\|_{p_{s}^{*}, K}^{p_{s}^{*}} \\
\geq & {\left[a\left(\frac{1}{\theta p}-\frac{1}{q}\right)-\frac{\gamma^{+}}{H}\left(\frac{1}{p}-\frac{1}{q}\right)\right]\left\|u_{n}\right\|^{p}+\left(\frac{1}{q}-\frac{1}{p_{s}^{*}}\right)\left\|u_{n}^{+}\right\|_{p_{s}^{*}, K}^{p^{*}} . }
\end{aligned}
$$

Therefore, by (2.7) there exists $\sigma_{\gamma, \lambda}>0$ such that as $n \rightarrow \infty$

$$
\begin{gathered}
c_{\gamma, \lambda}+\sigma_{\gamma, \lambda}\left\|u_{n}\right\|+o(1) \geq \mu_{\gamma}\left\|u_{n}\right\|^{p}, \\
\mu_{\gamma}=a\left(\frac{1}{\theta p}-\frac{1}{\tau}\right)-\frac{\gamma^{+}}{H}\left(\frac{1}{p}-\frac{1}{\tau}\right)>0,
\end{gathered}
$$

since $\gamma<\kappa H$, where $\kappa$ is given in (1.5). Therefore, $\left(u_{n}\right)_{n}$ is bounded in $E$. By the uniform convexity of the space $E$, Lemma 2.1, and (1.4), 
there exists $u_{\gamma, \lambda} \in E$ such that, going if necessary to a subsequence,

$$
\begin{array}{ll}
u_{n} \rightarrow u_{\gamma, \lambda} \text { in } E, & \left\|u_{n}\right\| \rightarrow \alpha_{\gamma, \lambda}, \\
u_{n} \rightarrow u_{\gamma, \lambda} \text { in } L^{p_{s}^{*}}\left(\mathbb{R}^{N}, K\right), & u_{n} \rightarrow u_{\gamma, \lambda} \text { in } L^{p}\left(\mathbb{R}^{N},|x|^{-p s}\right), \\
\left\|u_{n}-u_{\gamma, \lambda}\right\|_{H} \rightarrow v_{\gamma, \lambda}, & u_{n} \rightarrow u_{\gamma, \lambda} \text { a.e. in } \mathbb{R}^{N}, \\
u_{n}^{+} \rightarrow u_{\gamma, \lambda}^{+} \text {a.e. in } \mathbb{R}^{N}, & u_{n}^{-} \rightarrow u_{\gamma, \lambda}^{-} \text {a.e. in } \mathbb{R}^{N},
\end{array}
$$

since $\left|u_{n}^{+}(x)-u_{\gamma, \lambda}^{+}(x)\right| \leq\left|u_{n}(x)-u_{\gamma, \lambda}(x)\right|$ and similarly $\left|u_{n}^{-}(x)-u_{\gamma, \lambda}^{-}(x)\right| \leq$ $\left|u_{n}(x)-u_{\gamma, \lambda}(x)\right|$ for a.a. $x \in \mathbb{R}^{N}$. Therefore, by (2.10) and the uniqueness of the weak limit, we deduce at once that

$$
u_{n}^{+} \rightarrow u_{\gamma, \lambda}^{+} \text {in } L^{p_{s}^{*}}\left(\mathbb{R}^{N}, K\right) \text { and } u_{n}^{-} \rightarrow u_{\gamma, \lambda}^{-} \text {in } L^{p_{s}^{*}}\left(\mathbb{R}^{N}, K\right),
$$

going to a further subsequence, if necessary.

Turning to (2.7), we have shown that

$$
c_{\gamma, \lambda}+o(1) \geq \mu_{\gamma}\left\|u_{n}\right\|^{p}+\left(\frac{1}{\tau}-\frac{1}{p_{s}^{*}}\right)\left\|u_{n}^{+}\right\|_{p_{s}^{*}, K}^{p_{s}^{*}},
$$

where $\mu_{\gamma}$ is given in (2.9) and $\tau$ in (1.5).

First, we assert that

$$
\lim _{\lambda \rightarrow \infty} \alpha_{\gamma, \lambda}=0
$$

Otherwise, $\lim \sup _{\lambda \rightarrow \infty} \alpha_{\gamma, \lambda}=\alpha_{\gamma}>0$. Hence there is a sequence $\left(\lambda_{k}\right)_{k}$, with $\lambda_{k} \uparrow \infty$ such that $\alpha_{\gamma, \lambda_{k}} \rightarrow \alpha_{\gamma}$ as $k \rightarrow \infty$. Then, letting $k \rightarrow \infty$ we get from (2.12) and Lemma 2.3 that

$$
0 \geq \mu_{\gamma} \alpha_{\gamma}^{p}>0
$$

This contradiction proves the assertion (2.13). Moreover, $\left\|u_{\gamma, \lambda}\right\| \leq \alpha_{\gamma, \lambda}$, since $u_{n} \rightarrow u_{\gamma, \lambda}$, and so $(K),(1.4)$, and (2.13) implies that

(2.14) $\lim _{\lambda \rightarrow \infty}\left\|u_{\gamma, \lambda}^{+}\right\|_{p_{s}^{*}, K}=\lim _{\lambda \rightarrow \infty}\left\|u_{\gamma, \lambda}\right\|_{p_{s}^{*}, K}=\lim _{\lambda \rightarrow \infty}\left\|u_{\gamma, \lambda}\right\|_{H}=\lim _{\lambda \rightarrow \infty}\left\|u_{\gamma, \lambda}\right\|=0$.

Let us prove that $\left(u_{n}\right)_{n}$, up to a possibly further beyond subsequence, converges strongly to $u_{\gamma, \lambda}$ in $E$. As shown in the proof of Lemma 2.4 of $[\mathbf{1 0}]$, by $(2.10)$ the sequence $\left(\mathcal{U}_{n}\right)_{n}$, defined in $\mathbb{R}^{2 N} \backslash \operatorname{Diag}\left(\mathbb{R}^{2 N}\right)$ by

$$
(x, y) \mapsto \mathcal{U}_{n}(x, y)=\frac{\left|u_{n}(x)-u_{n}(y)\right|^{p-2}\left[u_{n}(x)-u_{n}(y)\right]}{|x-y|^{(N+p s) / p^{\prime}}},
$$

is bounded in $L^{p^{\prime}}\left(\mathbb{R}^{2 N}\right)$ as well as $\mathcal{U}_{n} \rightarrow \mathcal{U}_{\gamma, \lambda}$ a.e. in $\mathbb{R}^{2 N}$, where

$$
\mathcal{U}_{\gamma, \lambda}(x, y)=\frac{\left|u_{\gamma, \lambda}(x)-u_{\gamma, \lambda}(y)\right|^{p-2}\left[u_{\gamma, \lambda}(x)-u_{\gamma, \lambda}(y)\right]}{|x-y|^{(N+p s) / p^{\prime}}} .
$$


Thus, going if necessary to a further subsequence, we get that $\mathcal{U}_{n} \rightarrow \mathcal{U}_{\gamma, \lambda}$ in $L^{p^{\prime}}\left(\mathbb{R}^{2 N}\right)$ as $n \rightarrow \infty$. Furthermore, $\left|u_{n}\right|^{p-2} u_{n} \rightarrow\left|u_{\gamma, \lambda}\right|^{p-2} u_{\gamma, \lambda}$ in $L^{p^{\prime}}\left(\mathbb{R}^{N}, V\right)$ by Proposition A.8 of $[\mathbf{3}]$. Hence,

$$
\left\langle u_{n}, \varphi\right\rangle \rightarrow\left\langle u_{\gamma, \lambda}, \varphi\right\rangle
$$

for any $\varphi \in E$, since $(x, y) \mapsto|\varphi(x)-\varphi(y)| \cdot|x-y|^{-(N+p s) / p} \in L^{p}\left(\mathbb{R}^{2 N}\right)$ and $\varphi \in L^{p}\left(\mathbb{R}^{N}, V\right)$. Similarly, by $(2.10),(2.11)$, and by Proposition A.8 of $[\mathbf{3}]$ we deduce that $\left|u_{n}\right|^{p_{s}^{*}-2} u_{n} \rightarrow\left|u_{\gamma, \lambda}\right|^{p_{s}^{*}-2} u_{\gamma, \lambda}$ in $L^{p_{s}^{* \prime}}\left(\mathbb{R}^{N}, K\right)$ and $\left(u_{n}^{+}\right)^{p_{s}^{*}-1} \rightarrow\left(u_{\gamma, \lambda}^{+}\right)^{p_{s}^{*}-1}$ in $L^{p_{s}^{* \prime}}\left(\mathbb{R}^{N}, K\right)$. In particular,

$$
\left\langle u_{n}^{+}, \varphi\right\rangle_{p_{s}^{*}, K} \rightarrow\left\langle u_{\gamma, \lambda}^{+}, \varphi\right\rangle_{p_{s}^{*}, K}
$$

as $n \rightarrow \infty$.

By Lemma 4.2 of [10] we get

$$
\begin{gathered}
\lim _{n \rightarrow \infty} \int_{\mathbb{R}^{N}}\left(f\left(x, u_{n}\right)-f\left(x, u_{\gamma, \lambda}\right)\right)\left(u_{n}-u_{\gamma, \lambda}\right) d x=0, \\
\lim _{n \rightarrow \infty} \int_{\mathbb{R}^{N}} f\left(x, u_{n}\right) \varphi d x=\int_{\mathbb{R}^{N}} f\left(x, u_{\gamma, \lambda}\right) \varphi d x
\end{gathered}
$$

for all $\varphi \in E$.

On the other hand, Theorem 2.1 of $[\mathbf{3 6}]$ and $(\mathcal{V})$ imply that

$$
u_{n} \rightarrow u_{\gamma, \lambda} \text { in } L^{\theta p}\left(\mathbb{R}^{N}\right) \text { and in } L^{r}\left(\mathbb{R}^{N}\right),
$$

since $p \leq \theta p<r<p_{s}^{*}$ by $(\mathcal{G})$. Furthermore, using $(\mathcal{G})$, with $\varepsilon=1 / \theta p$, we have

$$
|g(x, t)| \leq|t|^{\theta p-1}+K_{r}|t|^{r-1} \quad \text { for a.a. } x \in \mathbb{R}^{N} \text { and all } t \in \mathbb{R},
$$

where $K_{r}=r C_{1 / \theta p}$. Then, the Hölder inequality, (2.18), and (2.19) yield

$$
\begin{aligned}
\left|\int_{\mathbb{R}^{N}}\left(g\left(x, u_{n}\right)-g\left(x, u_{\gamma, \lambda}\right)\right)\left(u_{n}-u_{\gamma, \lambda}\right) d x\right| \\
\leq\left(\left\|u_{n}\right\|_{\theta p}^{\theta p-1}+\left\|u_{\gamma, \lambda}\right\|_{\theta p}^{\theta p-1}\right)\left\|u_{n}-u_{\gamma, \lambda}\right\|_{\theta p} \\
\quad+K_{r}\left(\left\|u_{n}\right\|_{r}^{r-1}+\left\|u_{\gamma, \lambda}\right\|_{r}^{r-1}\right)\left\|u_{n}-u_{\gamma, \lambda}\right\|_{r}=o(1)
\end{aligned}
$$

as $n \rightarrow \infty$. Similarly,

$$
\lim _{n \rightarrow \infty} \int_{\mathbb{R}^{N}} g\left(x, u_{n}\right) \varphi d x=\int_{\mathbb{R}^{N}} g\left(x, u_{\gamma, \lambda}\right) \varphi d x
$$

for all $\varphi \in E$. 
Consequently, (2.10), (2.15)-(2.17), and (2.21) give at once that $u_{\gamma, \lambda}$ satisfies the identity

$$
\begin{aligned}
& M\left(\alpha_{\gamma, \lambda}^{p}\right)\left\langle u_{\gamma, \lambda}, \varphi\right\rangle-\gamma\left\langle u_{\gamma, \lambda}, \varphi\right\rangle_{H} \\
&=\lambda \int_{\mathbb{R}^{N}} f\left(x, u_{\gamma, \lambda}\right) \varphi d x+\int_{\mathbb{R}^{N}} g\left(x, u_{\gamma, \lambda}\right) \varphi d x+\left\langle u_{\gamma, \lambda}^{+}, \varphi\right\rangle_{p_{s}^{*}, K}
\end{aligned}
$$

for any $\varphi \in E$. Hence, $u_{\gamma, \lambda}$ is a critical point of the $C^{1}(E)$ functional

$$
\begin{aligned}
J_{\alpha_{\gamma, \lambda}}(u)=\frac{1}{p} M\left(\alpha_{\gamma, \lambda}^{p}\right)\|u\|^{p} & -\frac{\gamma}{p}\|u\|_{H}^{p}-\lambda \int_{\mathbb{R}^{N}} F(x, u) d x \\
& -\int_{\mathbb{R}^{N}} G(x, u) d x-\frac{1}{p_{s}^{*}}\left\|u^{+}\right\|_{p_{s}^{*}, K}^{p^{*}} .
\end{aligned}
$$

By the Hölder inequality we have

$$
|\langle u, v\rangle| \leq\|u\|^{p-1}\|v\| \quad \text { for all } u, v \in E
$$

and so, for any $u \in E$ the functional $\langle u, \cdot\rangle$ is linear and continuous on $E$. Consequently, (2.7), (2.10), (2.11), and (2.22) give as $n \rightarrow \infty$

$$
\begin{aligned}
o(1)= & \left\langle J_{\gamma, \lambda}^{\prime}\left(u_{n}\right)-J_{\alpha_{\gamma, \lambda}}^{\prime}\left(u_{\gamma, \lambda}\right), u_{n}-u_{\gamma, \lambda}\right\rangle_{E^{\prime}, E} \\
= & M\left(\left\|u_{n}\right\|^{p}\right)\left\|u_{n}\right\|^{p}+M\left(\alpha_{\gamma, \lambda}^{p}\right)\left\|u_{\gamma, \lambda}\right\|^{p} \\
& -M\left(\left\|u_{n}\right\|^{p}\right)\left\langle u_{n}, u_{\gamma, \lambda}\right\rangle-M\left(\alpha_{\gamma, \lambda}^{p}\right)\left\langle u_{\gamma, \lambda}, u_{n}\right\rangle \\
& -\gamma\left\langle u_{n}-u_{\gamma, \lambda}, u_{n}-u_{\gamma, \lambda}\right\rangle_{H} \\
& -\left\langle u_{n}-u_{\gamma, \lambda}, u_{n}-u_{\gamma, \lambda}\right\rangle_{p_{s}^{*}, K}+o(1) \\
= & M\left(\alpha_{\gamma, \lambda}^{p}\right)\left(\alpha_{\gamma, \lambda}^{p}-\left\|u_{\gamma, \lambda}\right\|^{p}\right)-\gamma\left\|u_{n}\right\|_{H}^{p}+\gamma\left\|u_{\gamma, \lambda}\right\|_{H}^{p} \\
& -\left\|u_{n}^{+}\right\|_{p_{s}^{*}, K}^{p_{s}^{*}}+\left\|u_{\gamma, \lambda}^{+}\right\|_{p_{s}^{*}, K}^{p_{s}^{*}}+o(1),
\end{aligned}
$$

where

$$
\begin{aligned}
\left\langle u_{n}-u_{\gamma, \lambda}, u_{n}-u_{\gamma, \lambda}\right\rangle_{H} & =\int_{\mathbb{R}^{N}} \frac{\left(\left|u_{n}\right|^{p-2} u_{n}-\left|u_{\gamma, \lambda}\right|^{p-2} u_{\gamma, \lambda}\right)\left(u_{n}-u_{\gamma, \lambda}\right)}{|x|^{p s}} d x, \\
\left\langle u_{n}-u_{\gamma, \lambda}, u_{n}-u_{\gamma, \lambda}\right\rangle_{p_{s}^{*}, K} & =\int_{\mathbb{R}^{N}} K(x)\left[\left(u_{n}^{+}\right)^{p_{s}^{*}-1}-\left(u_{\gamma, \lambda}^{+}\right)^{p_{s}^{*}-1}\right]\left(u_{n}-u_{\gamma, \lambda}\right) d x
\end{aligned}
$$


since $\left\|u_{n}\right\| \rightarrow \alpha_{\gamma, \lambda}$ as $n \rightarrow \infty$, as declared in (2.10). Furthermore, using again $(2.10),(2.11)$, and the celebrated Brézis and Lieb lemma of [7]

$$
\begin{aligned}
\left\|u_{n}\right\|^{p} & =\left\|u_{n}-u_{\gamma, \lambda}\right\|^{p}+\left\|u_{\gamma, \lambda}\right\|^{p}+o(1), \\
\left\|u_{n}\right\|_{H}^{p} & =\left\|u_{n}-u_{\gamma, \lambda}\right\|_{H}^{p}+\left\|u_{\gamma, \lambda}\right\|_{H}^{p}+o(1), \\
\left\|u_{n}^{+}\right\|_{p_{s}^{*}, K}^{p_{*}^{*}} & =\left\|u_{n}^{+}-u_{\gamma, \lambda}^{+}\right\|_{p_{s}^{*}, K}^{p_{*}^{*}}+\left\|u_{\gamma, \lambda}^{+}\right\|_{p_{s}^{*}, K}^{p^{*}}+o(1)
\end{aligned}
$$

as $n \rightarrow \infty$. Hence, from (2.23) we derive that as $n \rightarrow \infty$ (2.25) $M\left(\alpha_{\gamma, \lambda}^{p}\right)\left\|u_{n}-u_{\gamma, \lambda}\right\|^{p}-\gamma\left\|u_{n}-u_{\gamma, \lambda}\right\|_{H}^{p}-\left\|u_{n}^{+}-u_{\gamma, \lambda}^{+}\right\|_{p_{s}^{*}, K}^{p_{*}^{*}}=o(1)$.

Now, passing if necessary to a further subsequence, still called $\left(u_{n}\right)_{n}$, there exists a number $\ell_{\gamma, \lambda}$ such that

$$
\left\|u_{n}^{+}-u_{\gamma, \lambda}^{+}\right\|_{p_{s}^{*}, K} \rightarrow \ell_{\gamma, \lambda}
$$

as $n \rightarrow \infty$. Hence, using the notation in (2.10) and (2.26), we have obtained the main formula

$$
\begin{aligned}
M\left(\alpha_{\gamma, \lambda}^{p}\right) \lim _{n \rightarrow \infty}\left\|u_{n}-u_{\gamma, \lambda}\right\|^{p}= & \lim _{n \rightarrow \infty}\left\|u_{n}^{+}-u_{\gamma, \lambda}^{+}\right\|_{p_{s}^{*}, K}^{p_{*}^{*}} \\
& +\gamma \lim _{n \rightarrow \infty}\left\|u_{n}-u_{\gamma, \lambda}\right\|_{H}^{p}=\ell_{\gamma, \lambda}^{p_{s}^{*}}+\gamma v_{\gamma, \lambda}^{p} .
\end{aligned}
$$

Let us divide the proof in two parts.

Case $\|K\|_{\infty}=0$. Clearly $\ell_{\gamma, \lambda}=0$ in (2.26) and (2.27). Assume for contradiction that $\imath_{\gamma, \lambda}>0$. Then, from (1.4) and (2.27)

$$
\begin{aligned}
M\left(\alpha_{\gamma, \lambda}^{p}\right) \lim _{n \rightarrow \infty}\left\|u_{n}-u_{\gamma, \lambda}\right\|^{p} & =\gamma \lim _{n \rightarrow \infty}\left\|u_{n}-u_{\gamma, \lambda}\right\|_{H}^{p}<a H \lim _{n \rightarrow \infty}\left\|u_{n}-u_{\gamma, \lambda}\right\|_{H}^{p} \\
& \leq M\left(\alpha_{\gamma, \lambda}^{p}\right) \lim _{n \rightarrow \infty}\left\|u_{n}-u_{\gamma, \lambda}\right\|^{p},
\end{aligned}
$$

which is impossible. Hence, $\imath_{\gamma, \lambda}=0$ for all $\lambda>0$. Thus, using also (2.27) and the fact that $\ell_{\gamma, \lambda}=0$, we get

$$
\lim _{n \rightarrow \infty}\left\|u_{n}-u_{\gamma, \lambda}\right\|=\lim _{n \rightarrow \infty}\left\|u_{n}-u_{\gamma, \lambda}\right\|_{H}=0
$$

by (1.3). In conclusion, $u_{n} \rightarrow u_{\gamma, \lambda}$ in $E$ as $n \rightarrow \infty$ for all $\lambda>0$ as required.

Case $\|K\|_{\infty}>0$. By (2.12) and (2.24), we obtain as $n \rightarrow \infty$

$$
\begin{aligned}
c_{\gamma, \lambda}+o(1) & \geq\left(\frac{1}{\tau}-\frac{1}{p_{s}^{*}}\right)\left\|u_{n}^{+}\right\|_{p_{s}^{*}, K}^{p_{*}^{*}}=\left(\frac{1}{\tau}-\frac{1}{p_{s}^{*}}\right)\left[\ell_{\gamma, \lambda}^{p_{s}^{*}}+\left\|u_{\gamma, \lambda}^{+}\right\|_{p_{s}^{*}, K}^{p_{s}^{*}}\right]+o(1) \\
& \geq\left(\frac{1}{\tau}-\frac{1}{p_{s}^{*}}\right) \ell_{\gamma, \lambda}^{p_{s}^{*}}+o(1) .
\end{aligned}
$$


Then, Lemma 2.3 implies that

$$
\lim _{\lambda \rightarrow \infty} \ell_{\gamma, \lambda}=0 \text {. }
$$

Since $\gamma<a H$ there exists $c \in[0,1)$ such that $\gamma^{+}=c a H$. Of course, (2.27) can be rewritten as

$(1-c) M\left(\alpha_{\gamma, \lambda}^{p}\right) \lim _{n \rightarrow \infty}\left\|u_{n}-u_{\gamma, \lambda}\right\|^{p}+c M\left(\alpha_{\gamma, \lambda}^{p}\right) \lim _{n \rightarrow \infty}\left\|u_{n}-u_{\gamma, \lambda}\right\|^{p}=\ell_{\gamma, \lambda}^{p_{s}^{*}}+\gamma \imath_{\gamma, \lambda}^{p}$.

Thus, $\ell_{\gamma, \lambda}^{p_{s}^{*}}+\gamma^{+} \imath_{\gamma, \lambda}^{p} \geq(1-c) S\|K\|_{\infty}^{-p / p_{s}^{*}} a \ell_{\gamma, \lambda}^{p}+c a H \imath_{\gamma, \lambda}^{p}$ for all $\lambda>0$ by $(K),(1.3)$, and (1.4), being $c \in[0,1)$. Therefore, since $\gamma^{+}=c a H$,

$$
\ell_{\gamma, \lambda}^{p_{s}^{*}} \geq(1-c) S\|K\|_{\infty}^{-p / p_{s}^{*}} a \ell_{\gamma, \lambda}^{p} .
$$

Consequently, (2.28) and (2.29) imply at once that there exists a threshold $\lambda^{*}=\lambda^{*}(\gamma)>0$ such that $\ell_{\gamma, \lambda}=0$ for all $\lambda \geq \lambda^{*}$. In other words,

$$
\lim _{n \rightarrow \infty}\left\|u_{n}^{+}-u_{\gamma, \lambda}^{+}\right\|_{p_{s}^{*}, K}=0
$$

for all $\lambda \geq \lambda^{*}$. From now on we can proceed as in the first case, and prove that $\imath_{\gamma, \lambda}=0$ for all $\lambda \geq \lambda^{*}$. Hence, using also (2.27) and (1.3), we get $u_{n} \rightarrow u_{\gamma, \lambda}$ in $E$ as $n \rightarrow \infty$ for all $\lambda \geq \lambda^{*}$ as required. This completes the proof.

As already noted in the introduction, besides the obvious case $M \equiv a$, in which $\kappa=a$, there are several non monotone Kirchhoff functions $M$ for which $\kappa=a$, that is $\theta=1$. We refer to [10] for specific simple examples. We also point out that in the proof of the main Lemma 2.4 we use $(\mathcal{V})$ only to get $(2.20)$ and $(2.21)$. Therefore, if $g \equiv 0$, the assertion of Lemma 2.4 continues to hold under the sole assumption $(V)$ on the potential $V$.

Proof of Theorem 1.1: Fix $\gamma \in(-\infty, \kappa H)$. Thanks to Lemmas 2.2 and 2.4 the functional $J_{\gamma, \lambda}$ satisfies all the assumptions of the mountain pass theorem for any $\lambda>0$ when $\|K\|_{\infty}=0$ and for any $\lambda \geq \lambda^{*}$, with $\lambda^{*}=\lambda^{*}(\gamma)>0$, if $\|K\|_{\infty}>0$. This guarantees the existence of a critical point $u_{\gamma, \lambda} \in E$ for $J_{\gamma, \lambda}$ at level $c_{\gamma, \lambda}$. Since $J_{\gamma, \lambda}\left(u_{\gamma, \lambda}\right)=$ $c_{\gamma, \lambda}>0=J_{\gamma, \lambda}(0)$ we have that $u_{\gamma, \lambda} \neq 0$. Moreover the asymptotic behavior (1.6) follows directly from (2.14).

Finally, assume that $f, g$ are Carathéodory functions, satisfying (1.7), and that $h \in L^{\nu^{\prime}}\left(\mathbb{R}^{N}\right)$ is nonnegative in $\mathbb{R}^{N}$, with $\nu^{\prime}=\nu /(\nu-1)$ and $\nu \in\left[p, p_{s}^{*}\right]$. Let $\gamma<a H$ and $\lambda \geq 0$ be fixed. Let $u$ be a solution of (1.8) in $E$. Put $u=u^{+}-u^{-}$. It is not hard to show that for a.a. $x, y \in \mathbb{R}^{N}$

$$
\operatorname{sign}[u(x)-u(y)] \cdot\left[u^{-}(x)-u^{-}(y)\right]=-\left|u^{-}(x)-u^{-}(y)\right|
$$


and, as noted in the proof of Lemma 2.4, both $u^{+}$and $u^{-}$are still in $E$. Combining these facts and recalling that $\left|u^{-}(x)-u^{-}(y)\right| \leq|u(x)-u(y)|$ for a.a. $x, y \in \mathbb{R}^{N}$, we get at once that

$$
\begin{aligned}
\left\langle u, u^{-}\right\rangle_{s, p} & =\iint_{\mathbb{R}^{2 N}} \frac{|u(x)-u(y)|^{p-1} \operatorname{sign}[u(x)-u(y)] \cdot\left[u^{-}(x)-u^{-}(y)\right]}{|x-y|^{N+p s}} d x d y \\
& \leq-\left[u^{-}\right]_{s, p}^{p} .
\end{aligned}
$$

Similarly, $\left\langle u, u^{-}\right\rangle_{V} \leq-\left\|u^{-}\right\|_{p, V}^{p}$. In conclusion, $\left\langle u, u^{-}\right\rangle \leq-\left\|u^{-}\right\|^{p}$. Thus, by the definition of solution for (1.8), taking as test function $\varphi=$ $u^{-} \in E$, we have

$$
\begin{aligned}
-a\left\|u^{-}\right\|^{p} & \geq-M\left(\|u\|^{p}\right)\left\|u^{-}\right\|^{p} \\
& \geq M\left(\|u\|^{p}\right)\left\langle u, u^{-}\right\rangle \geq-\gamma\left\|u^{-}\right\|_{H}^{p}+\int_{\mathbb{R}^{N}} h(x) u^{-} d x \geq-\frac{\gamma^{+}}{H}\left\|u^{-}\right\|^{p}
\end{aligned}
$$

by (1.3), (1.4), (1.7), and the fact that $h \geq 0$ a.e. in $\mathbb{R}^{N}$. Hence, $\left\|u^{-}\right\|=0$, since $\gamma<a H$. In conclusion, $u^{-}=0$ a.e. in $\mathbb{R}^{N}$ and so $u$ is nonnegative in $\mathbb{R}^{N}$, as required.

From the proofs of Lemmas $2.2-2.4$ it is evident that in condition $(\mathcal{F})-\left(f_{1}\right)$ the function $(x, t) \mapsto w(x)\left(t^{+}\right)^{q-1}$ can be replaced by $(x, t) \mapsto w(x)|t|^{q-2} t$, and similarly $(x, t) \mapsto K(x)\left(t^{+}\right)^{p_{s}^{*}-1}$ by $(x, t) \mapsto$ $K(x)|t|^{p_{s}^{*}-2} t$. Thus existence of a nontrivial mountain pass solution of

$$
\begin{aligned}
& M\left(\|u\|^{p}\right) \cdot\left[(-\Delta)_{p}^{s} u+V(x)|u|^{p-2} u\right]-\gamma \frac{|u|^{p-2} u}{|x|^{p s}} \\
&=\lambda f(x, u)+g(x, u)+K(x)|u|^{p_{s}^{*}-2} u
\end{aligned}
$$

in $\mathbb{R}^{N}$, as well as the validity of (1.6), can be obtained in a similar way. Of course, we cannot conclude any longer about its sign.

In this section we prove the main existence result for (1.1) in the radial case. To apply the mountain pass theorem and the Ekeland variational principle, we need the following embedding result obtained combining Theorem II.1 of [28] with Lemma 2.1.

Lemma 2.5. Let $N \geq 2$. For any $p<\nu<p_{s}^{*}$, the embedding $E_{\mathrm{rad}} \hookrightarrow$ $L^{\nu}\left(\mathbb{R}^{N}\right)$ is compact, where

$$
E_{\mathrm{rad}}=\{u \in E: u \text { is radially symmetric with respect to } 0\} .
$$

In order to avoid condition $(\mathcal{V})$ we pass into the radial setting. In order to get the compactness of the embedding $E \hookrightarrow L^{\nu}\left(\mathbb{R}^{N}\right), p<\nu<p_{s}^{*}$, we 
need to restrict the study searching solutions of (1.1) in $E_{\text {rad }}$, where

$$
E_{\mathrm{rad}}=\{u \in E: u \text { is radially symmetric with respect to } 0\} .
$$

Thus, until the end of the section $K, V, f$, and $g$ are assumed to be radially symmetric functions in $x$, and that $(\mathcal{M}),(V),(\mathcal{F})$, and $(\mathcal{G})$ hold, without further mentioning. Again, the geometry stated in Lemma 2.2 continues to hold. Therefore, for any $\gamma<a H$ and $\lambda>0$ we now put

$$
\begin{gathered}
c_{\gamma, \lambda}=\inf _{\xi \in \Gamma} \max _{t \in[0,1]} J_{\gamma, \lambda}(\xi(t)), \\
\Gamma=\left\{\xi \in C\left([0,1], E_{\mathrm{rad}}\right): \xi(0)=0, \xi(1)=e\right\},
\end{gathered}
$$

where $e \in E_{\mathrm{rad}}$ is the function constructed in Lemma 2.2. Then Lemma 2.3 continues to hold without significant adjustments. The notable changes now occur in the proof of Lemma 2.4 .

Lemma 2.6. Let $N \geq 2$ and let $\gamma \in(-\infty, \kappa H)$ be fixed. If $\|K\|_{\infty}=0$, then $J_{\gamma, \lambda}$ satisfies the Palais-Smale condition in $E_{\mathrm{rad}}$ at level $c_{\gamma, \lambda}$ for all $\lambda>0$. While if $\|K\|_{\infty}>0$, then there exists $\lambda^{*}=\lambda^{*}(\gamma)>0$ such that $J_{\gamma, \lambda}$ satisfies the Palais-Smale condition at level $c_{\gamma, \lambda}$ for any $\lambda \geq \lambda^{*}$.

Proof: Fix $\gamma<\kappa H, \lambda>0$, and let $\left(u_{n}\right)_{n} \subset E_{\text {rad }}$ be a Palais-Smale sequence of $J_{\gamma, \lambda}$ at level $c_{\gamma, \lambda}$. Then we can proceed exactly as in the proof of Lemma 2.4, replacing now Theorem 2.1 of $[\mathbf{3 6}]$ and $(\mathcal{V})$ by Theorem II.1 of [28], combined with Lemma 2.1, in order to get (2.20) and $(2.21)$, since $1<p \leq \theta p<\min \{q, \mu\} \leq \max \{q, \mu\}<p_{s}^{*}$ by $(\mathcal{M})$, $(\mathcal{F})$, and $(\mathcal{G})$. Indeed, Theorem II.1 of $[\mathbf{2 8}]$ and Lemma 2.1 yield that for any exponent $\nu$, with $p<\nu<p_{s}^{*}$, the embedding $E_{\mathrm{rad}} \hookrightarrow L^{\nu}\left(\mathbb{R}^{N}\right)$ is compact. The rest of the proof remains unchanged.

Proof of Theorem 1.2: Fix $\gamma \in(-\infty, \kappa H)$. By Lemmas 2.2 and 2.6 the functional $J_{\gamma, \lambda}$ satisfies all the assumptions of the mountain pass theorem in $E_{\text {rad }}$ for any $\lambda>0$ when $\|K\|_{\infty}=0$ and for any $\lambda \geq \lambda^{*}$, with $\lambda^{*}=\lambda^{*}(\gamma)>0$, if $\|K\|_{\infty}>0$. This guarantees the existence of a critical point $u_{\gamma, \lambda} \in E_{\mathrm{rad}}$ for $J_{\gamma, \lambda}$ at level $c_{\gamma, \lambda}$. Since $J_{\gamma, \lambda}\left(u_{\gamma, \lambda}\right)=c_{\gamma, \lambda}>0=$ $J_{\gamma, \lambda}(0)$ we have that $u_{\gamma, \lambda} \neq 0$. Moreover the asymptotic behavior (1.6) follows directly from $(2.14)$.

Up to this moment, the function $u_{\gamma, \lambda}$ is a solution of (1.1) only in the $E_{\text {rad }}$ sense. Let us show that $u_{\gamma, \lambda}$ is a solution of (1.1) in the whole space $E$, that is in sense of definition (2.2).

To this aim we use a version of the well known principle of symmetric criticality, due to Palais in [34], in the form of Proposition 3.1 of [14], which holds in reflexive strictly convex Banach spaces as proved in Lemma 5.4 of $[\mathbf{1 0}]$. 
Let $S O(N)$ denote the special orthogonal group, that is

$$
S O(N)=\left\{A \in \mathbb{R}^{N \times N}: A^{t} A=I_{N} \text { and } \operatorname{det} A=1\right\} .
$$

Next, consider the following subgroup of linear operators of $E$ in itself

$$
G=\{a: E \rightarrow E: a u=u \circ A, \text { where } A \in S O(N)\} .
$$

Observe that $G$ is a subgroup of isometries of $E$. Indeed, fixed $u$ in $E$, for all $a \in G$

$$
\begin{aligned}
\|a u\|^{p} & =\iint_{\mathbb{R}^{2 N}} \frac{|u(A x)-u(A y)|^{p}}{|x-y|^{N+p s}} d x d y+\int_{\mathbb{R}^{N}} V(x)|u(A x)|^{p} d x \\
& =\iint_{\mathbb{R}^{2 N}} \frac{\left|u\left(x^{\prime}\right)-u\left(y^{\prime}\right)\right|^{p}}{\left|x^{\prime}-y^{\prime}\right|^{N+p s}} d x^{\prime} d y^{\prime}+\int_{\mathbb{R}^{N}} V\left(x^{\prime}\right)\left|u\left(x^{\prime}\right)\right|^{p} d x^{\prime}=\|u\|^{p},
\end{aligned}
$$

since $|x-y|=|A(x-y)|=|A x-A y|=\left|x^{\prime}-y^{\prime}\right|, \operatorname{det} A=1$, and since $V$ is a radial function. Furthermore, $E_{\mathrm{rad}}=\{u \in E: a u=u$ for all $a \in G\}$. To apply Lemma 5.4 of $[\mathbf{1 0}]$ to the functional $J_{\gamma, \lambda}$, we need to show that $J_{\gamma, \lambda} \circ a=J_{\gamma, \lambda}$ for all $a \in G$. Fixed $u \in E$, for all $a \in G$ we have

$$
\begin{aligned}
\left(J_{\gamma, \lambda} \circ a\right)(u)= & \frac{1}{p}\left(\mathscr{M}\left(\|a u\|^{p}\right)-\gamma\|a u\|_{H}^{p}\right)-\lambda \int_{\mathbb{R}^{N}} F(x, u(A x)) d x \\
& -\int_{\mathbb{R}^{N}} G(x, u(A x)) d x-\frac{1}{p_{s}^{*}}\left\|(a u)^{+}\right\|_{p_{s}^{*}, K}^{p^{*}} \\
= & \frac{1}{p}\left(\mathscr{M}\left(\|u\|^{p}\right)-\gamma\|u\|_{H}^{p}\right)-\lambda \int_{\mathbb{R}^{N}} F\left(x^{\prime}, u\left(x^{\prime}\right)\right) d x^{\prime} \\
& -\int_{\mathbb{R}^{N}} G\left(x^{\prime}, u\left(x^{\prime}\right)\right) d x^{\prime}-\frac{1}{p_{s}^{*}}\left\|u^{+}\right\|_{p_{s}^{*}, K}^{p^{*}} \\
= & J_{\gamma, \lambda}(u),
\end{aligned}
$$

since $K, V, f$, and $g$ are radial functions in $x$. Hence, $J_{\gamma, \lambda}$ satisfies Lemma 5.4 of $[\mathbf{1 0}]$.

Now, $u_{\gamma, \lambda}$ is critical point of $\left.J_{\gamma, \lambda}\right|_{E_{\text {rad }}}$, that is

$$
\left\langle J_{\gamma, \lambda}^{\prime}\left(u_{\gamma, \lambda}\right), \varphi\right\rangle_{\left(E_{\mathrm{rad}}\right)^{\prime}, E_{\mathrm{rad}}}=0 \quad \text { for any } \varphi \in E_{\mathrm{rad}} .
$$

Then, Lemma 5.4 of [10] implies that $u_{\gamma, \lambda}$ is a critical point of $J_{\gamma, \lambda}$ in the whole space $E$. Thus, $u_{\gamma, \lambda}$ is a solution of (1.1) in the sense of definition (2.2). 


\section{The Schrödinger-Kirchhoff equation (1.9)}

Throughout the section we assume that $(\mathcal{M})$ and $(V)$ hold, that $q \in$ $\left(\theta p, p_{s}^{*}\right)$, that the weight $w>0$ a.e. in $\mathbb{R}^{N}$ and of class $L^{\wp}\left(\mathbb{R}^{N}\right)$, with $\wp=p_{s}^{*} /\left(p_{s}^{*}-q\right)$. We also treat, for simplicity, the only interesting case where $K>0$ a.e. in $\mathbb{R}^{N}$. Problem (1.9) has a variational structure and the underlying functional is $J_{\gamma, \lambda}: E \rightarrow \mathbb{R}$, given by

$$
\begin{gathered}
J_{\gamma, \lambda}(u)=\frac{1}{p}\left[\mathscr{M}\left(\|u\|^{p}\right)-\gamma\|u\|_{H}^{p}\right]-\mathcal{H}_{\lambda}(u), \\
\mathcal{H}_{\lambda}(u)=\frac{\lambda}{q}\left\|u^{+}\right\|_{q, w}^{q}+\frac{1}{p_{s}^{*}}\left\|u^{+}\right\|_{p_{s}^{s}, K}^{p_{s}^{*}}+\int_{\mathbb{R}^{N}} h(x) u(x) d x .
\end{gathered}
$$

Clearly, $J_{\gamma, \lambda}$ is well-defined and of class $C^{1}(E)$. We first prove that the geometry of the mountain pass lemma is still preserved for (1.9), provided that the nonnegative perturbation $h$ is sufficiently small in the $\nu^{\prime}$-norm, as shown first in $[\mathbf{3 6}]$ and then in $[\mathbf{1 0}]$ for more general equations.

Lemma 3.1. Fix $\gamma \in(-\infty, a H)$ and $\lambda \geq 0$. Then, there exists a nonnegative radial function e in $C_{0}^{\infty}\left(\mathbb{R}^{N}\right)$, depending only on $\gamma^{-}$, but independent of $\lambda \geq 0$ and $h$, such that $\|e\| \geq 2$ and $J_{\gamma, \lambda}(e)<0$. Furthermore, there exist numbers $\alpha=\alpha\left(\gamma^{+}, \lambda\right)>0, \rho=\rho\left(\gamma^{+}, \lambda\right) \in(0,1]$, and $\delta=\delta\left(\gamma^{+}, \lambda\right)>0$ such that $J_{\gamma, \lambda}(u) \geq \alpha$ for all $u \in E$, with $\|u\|=\rho$, and for all $h \in L^{\nu^{\prime}}\left(\mathbb{R}^{N}\right)$, with $\|h\|_{\nu^{\prime}} \leq \delta$.

Moreover, for all parameters $\gamma \in(-\infty, a H), \lambda \geq 0$ and for all perturbations $h \in L^{\nu^{\prime}}\left(\mathbb{R}^{N}\right)$, with $0<\|h\|_{\nu^{\prime}} \leq \delta=\delta\left(\gamma^{+}, \lambda\right)$, there exist a sequence $\left(v_{n}\right)_{n}$ and some nonnegative function $u_{\gamma, \lambda}$ in $E$ such that for all $n$

$$
\begin{gathered}
\left\|v_{n}\right\|<\rho, \quad m_{\gamma, \lambda} \leq J_{\gamma, \lambda}\left(v_{n}\right) \leq m_{\gamma, \lambda}+\frac{1}{n}, \\
v_{n} \rightarrow u_{\gamma, \lambda} \in \bar{B}_{\rho}, \quad v_{n} \rightarrow u_{\gamma, \lambda} \text { a.e. in } \mathbb{R}^{N}, \quad \text { and } J_{\gamma, \lambda}^{\prime}\left(v_{n}\right) \rightarrow 0
\end{gathered}
$$

as $n \rightarrow \infty$, where

$$
m_{\gamma, \lambda}=\inf \left\{J_{\gamma, \lambda}(u): u \in \bar{B}_{\rho}\right\}<0 \quad \text { and } \quad B_{\rho}=\{u \in E:\|u\|<\rho\} .
$$

Finally, for all $\gamma \in(-\infty, a H)$ and $\lambda \geq 0$ any solution $u \in E$ of (1.9) is nonnegative in $\mathbb{R}^{N}$ for all nonnegative perturbation $h \in L^{\nu^{\prime}}\left(\mathbb{R}^{N}\right)$. 
Proof: Fix $\gamma<a H$ and $\lambda \geq 0$. Take a radial function $v \in C_{0}^{\infty}\left(\mathbb{R}^{N}\right)$, with $v \geq 0$ in $\mathbb{R}^{N}$ and $\|v\|=1$. By (2.3) we have for $t \rightarrow \infty$

$$
\begin{aligned}
J_{\gamma, \lambda}(t v) & \leq \frac{\mathscr{M}(1)}{p} t^{\theta p}-\frac{\gamma}{p}\|v\|_{H}^{p} t^{p}-\lambda \frac{\|v\|_{,, w}^{q}}{q} t^{q}-\frac{\|v\|_{p_{s}^{*}, K}^{p^{*}}}{p_{s}^{*}} t^{p_{s}^{*}}-t \int_{\mathbb{R}^{N}} h(x) v d x \\
& \leq \frac{\mathscr{M}(1)}{p} t^{\theta p}+\frac{\gamma^{-}}{p}\|v\|_{H}^{p} t^{p}-\frac{\|v\|_{p_{s}^{*}, K}^{p^{*}}}{p_{s}^{*}} t^{p_{s}^{*}} \rightarrow-\infty,
\end{aligned}
$$

since $\lambda \geq 0, h$, and $v$ are nonnegative in $\mathbb{R}^{N}, K>0$ a.e. in $\mathbb{R}^{N}$ and of course $p \leq \theta p<p_{s}^{*}$. Hence, taking $e=\tau_{0} v$, with $\tau_{0}>0$ sufficiently large, we obtain at once that $\|e\| \geq 2$ and $J_{\gamma, \lambda}(e)<0$. Clearly, $e$ depends on neither $\lambda$ nor $h$.

By Lemma 2.1, $(K),(1.3)$, and (1.4) there exists a positive constant $S_{K}$ such that for all $u \in E$

$$
\begin{aligned}
J_{\gamma, \lambda}(u) & \geq \frac{a}{p}\|u\|^{p}-\frac{\gamma}{p}\|u\|_{H}^{p}-\frac{\lambda}{q}\left\|u^{+}\right\|_{q, w}^{q}-\frac{1}{p_{s}^{*}}\left\|u^{+}\right\|_{p_{s}^{*}, K}^{p_{s}^{*}}-\int_{\mathbb{R}^{N}} h(x) u d x \\
& \geq\left(\frac{a}{p}-\frac{\gamma^{+}}{p H}\right)\|u\|^{p}-\frac{\lambda}{q} C_{w}^{q}\|u\|^{q}-S_{K}\|u\|^{p_{s}^{*}}-C_{\nu}\|h\|_{\nu^{\prime}}\|u\| .
\end{aligned}
$$

Setting

$$
\eta_{\gamma, \lambda}(t)=\left(\frac{a}{p}-\frac{\gamma^{+}}{p H}\right) t^{p-1}-\frac{\lambda}{q} C_{w}^{q} t^{q-1}-S_{K} t^{p_{s}^{*}-1} \quad \text { for all } t \in[0,1]
$$

we get the existence of $\rho=\rho\left(\gamma^{+}, \lambda\right) \in(0,1]$ such that $\max _{t \in[0,1]} \eta_{\gamma, \lambda}(t)=$ $\eta_{\gamma, \lambda}(\rho)>0$, since $p \leq \theta p<q<p_{s}^{*}$. Taking $\delta=\eta(\rho) / 2 C_{\nu}$, we obtain that $J_{\gamma, \lambda}(u) \geq \alpha=\rho \eta_{\gamma, \lambda}(\rho) / 2>0$ for all $u \in E$, with $\|u\|=\rho$, and for all perturbations $h \in L^{\nu^{\prime}}\left(\mathbb{R}^{N}\right)$, with $\|h\|_{\nu^{\prime}} \leq \delta$, as claimed.

Using the notation above, we fix $h \in L^{\nu^{\prime}}\left(\mathbb{R}^{N}\right)$, with $0<\|h\|_{\nu^{\prime}} \leq \delta$ and $h \geq 0$ a.e. in $\mathbb{R}^{N}$. First, we claim that there exists a nonnegative function $\psi \in C_{0}^{\infty}\left(\mathbb{R}^{N}\right)$ such that

$$
\int_{\mathbb{R}^{N}} h(x) \psi(x) d x>0 .
$$

Since $h \in L^{\nu^{\prime}}\left(\mathbb{R}^{N}\right) \backslash\{0\}$, and $h \geq 0$ a.e. in $\mathbb{R}^{N}$, the function

$$
\phi(x)=\left\{\begin{array}{ll}
h(x)^{\nu^{\prime}-1} & \text { if } h(x) \neq 0 \\
0 & \text { if } h(x)=0
\end{array} \in L^{\nu}\left(\mathbb{R}^{N}\right) .\right.
$$

Then, there exists a sequence $\left(h_{n}\right)_{n}$ in $C_{0}^{\infty}\left(\mathbb{R}^{N}\right)$ such that $h_{n} \rightarrow \phi$ strongly in $L^{\nu}\left(\mathbb{R}^{N}\right)$ and a.e. in $\mathbb{R}^{N}$, since $C_{0}^{\infty}\left(\mathbb{R}^{N}\right)$ is dense in $L^{\nu}\left(\mathbb{R}^{N}\right)$. 
Hence, for $n_{0} \in \mathbb{N}$ large enough we get

$$
h_{n_{0}} \geq 0 \text { a.e. in } \mathbb{R}^{N} \text { and }\left\|h_{n_{0}}-\phi\right\|_{\nu} \leq \frac{1}{2}\|h\|_{\nu^{\prime}}^{\nu^{\prime}-1} .
$$

Thus, by the Hölder inequality, we have

$$
\int_{\mathbb{R}^{N}} h_{n_{0}}(x) h(x) d x \geq-\left\|h_{n_{0}}-\phi\right\|_{\nu}\|h\|_{\nu^{\prime}}+\int_{\mathbb{R}^{N}}|h(x)|^{\nu^{\prime}} d x \geq \frac{1}{2}\|h\|_{\nu^{\prime}}^{\nu^{\prime}}>0,
$$

since $h \not \equiv 0$. The claim (3.3) is so proved, taking $\psi=h_{n_{0}}$.

Now, putting $M_{\rho}=\max _{\xi \in[0, \rho]} M\left(\xi^{p}\right)$, where $\rho=\rho\left(\gamma^{+}, \lambda\right)>0$ is the number given in the previous part of the lemma, by (3.3) we have

$$
\begin{aligned}
J_{\gamma, \lambda}(t \psi) \leq & \frac{1}{p} \mathscr{M}\left(\|t \psi\|^{p}\right)+\frac{\gamma^{-}\|\psi\|_{H}^{p}}{p} t^{p}-\frac{\lambda\left\|\psi^{+}\right\|_{q, w}^{q}}{q} t^{q} \\
& -\frac{\left\|\psi^{+}\right\|_{p_{s}^{*}, K}^{p^{*}}}{p_{s}^{*}} t^{p_{s}^{*}}-t \int_{\mathbb{R}^{N}} h(x) \psi d x \\
\leq & \frac{M_{\rho}\|\psi\|^{p}}{p} t^{p}+\frac{\gamma^{-}\|\psi\|_{H}^{p}}{p} t^{p}-\frac{\lambda\|\psi\|_{q, w}^{q}}{q} t^{q} \\
& -\frac{\|\psi\|_{p_{s}^{*}, K}^{p_{s}^{*}}}{p_{s}^{*}} t^{p_{s}^{*}}-t \int_{\mathbb{R}^{N}} h(x) \psi d x<0,
\end{aligned}
$$

for $t \in(0,1)$ small enough, since $1<p \leq \theta p<q<p_{s}^{*}$. Thus, we obtain that

$$
m_{\gamma, \lambda}=\inf \left\{J_{\gamma, \lambda}(u): u \in \bar{B}_{\rho}\right\}<0,
$$

where $B_{\rho}=\{u \in E:\|u\|<\rho\}$. Then, by the Ekeland variational principle in $\bar{B}_{\rho}$ and the first part of the lemma, there exists a sequence $\left(v_{n}\right)_{n} \subset$ $B_{\rho}$ such that

$$
m_{\gamma, \lambda} \leq J_{\gamma, \lambda}\left(v_{n}\right) \leq m_{\gamma, \lambda}+\frac{1}{n} \quad \text { and } \quad J_{\gamma, \lambda}(v) \geq J_{\gamma, \lambda}\left(v_{n}\right)-\frac{1}{n}\left\|v-v_{n}\right\|
$$

for all $n \in \mathbb{N}$ and for any $v \in \bar{B}_{\rho}$. Fixed $n \in \mathbb{N}$, for all $w \in S_{E}$, where $S_{E}=\{u \in E:\|u\|=1\}$, and for all $\sigma>0$ so small that $v_{n}+\sigma w \in \bar{B}_{\rho}$, we have

$$
J_{\gamma, \lambda}\left(v_{n}+\sigma w\right)-J_{\gamma, \lambda}\left(v_{n}\right) \geq-\frac{\sigma}{n}
$$

by (3.4). Since $J_{\gamma, \lambda}$ is Gâteaux differentiable in $E$, we get

$$
\left\langle J_{\gamma, \lambda}^{\prime}\left(v_{n}\right), w\right\rangle_{E^{\prime}, E}=\lim _{\sigma \rightarrow 0} \frac{J_{\gamma, \lambda}\left(v_{n}+\sigma w\right)-J_{\gamma, \lambda}\left(v_{n}\right)}{\sigma} \geq-\frac{1}{n}
$$


for all $w \in S_{E}$. Hence

$$
\left|\left\langle J_{\gamma, \lambda}^{\prime}\left(v_{n}\right), w\right\rangle_{E^{\prime}, E}\right| \leq \frac{1}{n}
$$

since $w \in S_{E}$ is arbitrary. Consequently, $J_{\gamma, \lambda}^{\prime}\left(v_{n}\right) \rightarrow 0$ in $E^{\prime}$ as $n \rightarrow \infty$ and clearly, up to a subsequence, the bounded sequence $\left(v_{n}\right)_{n}$ weakly converges to some $u_{\gamma, \lambda} \in \bar{B}_{\rho}$ and $v_{n} \rightarrow u_{\gamma, \lambda}$ a.e. in $\mathbb{R}^{N}$. Furthermore, we assume w.l.o.g. that $\left(v_{n}^{-}\right)_{n}$ weakly converges to $u_{\gamma, \lambda}^{-} \in \bar{B}_{\rho}$ in $E$ and $v_{n}^{-} \rightarrow u_{\gamma, \lambda}^{-}$a.e. in $\mathbb{R}^{N}$, as shown in the proof of Lemma 2.4. Moreover, by (1.3) and (1.4) as $n \rightarrow \infty$

$$
\begin{aligned}
o(1) & =-\left\langle J_{\gamma, \lambda}^{\prime}\left(v_{n}\right), v_{n}^{-}\right\rangle_{E^{\prime}, E}=-M\left(\left\|v_{n}\right\|^{p}\right)\left\langle v_{n}, v_{n}^{-}\right\rangle+\gamma\left\langle v_{n}, v_{n}^{-}\right\rangle_{H} \\
& \geq a\left\|v_{n}^{-}\right\|^{p}-\frac{\gamma^{+}}{H}\left\|v_{n}^{-}\right\|^{p}
\end{aligned}
$$

since $\left\langle v_{n}, v_{n}^{-}\right\rangle \leq-\left\|v_{n}^{-}\right\|^{p}$, the function $h \geq 0$ a.e. in $\mathbb{R}^{N}$ and $\gamma<a H$. Therefore, $\left(v_{n}^{-}\right)_{n}$ strongly converges to 0 in $E$ and $v_{n}^{-} \rightarrow 0$ a.e. in $\mathbb{R}^{N}$. Thus $u_{\gamma, \lambda}^{-}=0$ a.e. in $\mathbb{R}^{N}$. In particular, $u_{\gamma, \lambda} \geq 0$ in $\mathbb{R}^{N}$. This proves (3.1).

Finally, let $\lambda \geq 0$ be fixed and let $u$ be any solution of (1.9) in $E$. Following the argument of the last part of the proof of Theorem 1.1 and putting $u=u^{+}-u^{-}$, we have that both $u^{+}$and $u^{-}$are in $E$ and that $\left\langle u, u^{-}\right\rangle \leq-\left\|u^{-}\right\|^{p}$. Thus, by the definition of solution for (1.9), taking as test function $\varphi=u^{-} \in E$, we get by (1.3) and (1.4)

$$
\begin{aligned}
-a\left\|u^{-}\right\|^{p} & \geq-M\left(\|u\|^{p}\right)\left\|u^{-}\right\|^{p} \geq M\left(\|u\|^{p}\right)\left\langle u, u^{-}\right\rangle \\
& =-\gamma\left\|u^{-}\right\|_{H}^{p}+\int_{\mathbb{R}^{N}} h(x) u^{-} d x \geq-\frac{\gamma^{+}}{H}\left\|u^{-}\right\|^{p},
\end{aligned}
$$

since $h \geq 0$ a.e. in $\mathbb{R}^{N}$. Hence, $\left\|u^{-}\right\|=0$, that is $u^{-}=0$ a.e. in $\mathbb{R}^{N}$, since $\gamma<a H$. Thus $u$ is nonnegative in $\mathbb{R}^{N}$, as stated.

From the proof above it is evident that, in particular, $\|e\| \geq 2>\rho=$ $\rho\left(\gamma^{+}, \lambda\right)=\rho(\lambda)$ for all $\lambda>0$, whenever $\gamma \leq 0$. The next result takes inspiration of Theorem 1.3 of [30] and we assume that the Kirchhoff function $M$ is in the standard form (1.10).

Proof of Theorem 1.3. Fix $\gamma<a H$ and $\lambda>0$. Let us prove that $u_{\gamma, \lambda}$, given in (3.1) of Lemma 3.1, is actually in $B_{\rho}$, so that $u_{\gamma, \lambda}$ is a critical point of $J_{\gamma, \lambda}$ at level $m_{\gamma, \lambda}<0$. In other words, $u_{\gamma, \lambda}$ is a nontrivial nonnegative solution of (1.9). 
Clearly, $J_{\gamma, \lambda}\left(u_{\gamma, \lambda}\right) \geq m_{\gamma, \lambda}$, since $u_{\gamma, \lambda} \in \bar{B}_{\rho}$ by (3.1). Going if necessary to a subsequence, we may assume that

$$
\begin{gathered}
v_{n}^{+} \rightarrow u_{\gamma, \lambda} \text { in } L^{p_{s}^{*}}\left(\mathbb{R}^{N}, K\right), \quad v_{n}^{+} \rightarrow u_{\gamma, \lambda} \text { in } L^{p}\left(\mathbb{R}^{N},|x|^{-p s}\right), \\
\left\|v_{n}\right\| \rightarrow \alpha_{\gamma, \lambda}, \quad v_{n} \rightarrow u_{\gamma, \lambda} \text { in } L^{q}\left(\mathbb{R}^{N}, w\right)
\end{gathered}
$$

as $n \rightarrow \infty$ by Lemma 2.1 and the fact that $\left(v_{n}^{-}\right)_{n}$ strongly converges to zero in $E$, as shown in the proof of Lemma 3.1. Moreover, by (1.10) and (3.1) we have as $n \rightarrow \infty$

$$
\begin{aligned}
0= & \left\langle J_{\gamma, \lambda}^{\prime}\left(v_{n}\right), u_{\gamma, \lambda}\right\rangle_{E^{\prime}, E}+o(1)=\left(a+b \theta\left\|v_{n}\right\|^{p(\theta-1)}\right)\left\langle v_{n}, u_{\gamma, \lambda}\right\rangle \\
& -\gamma\left\langle v_{n}, u_{\gamma, \lambda}\right\rangle_{H}-\lambda\left\langle v_{n}^{+}, u_{\gamma, \lambda}\right\rangle_{q, w}-\left\langle v_{n}^{+}, u_{\gamma, \lambda}\right\rangle_{p_{s}^{*}, K} \\
& -\int_{\mathbb{R}^{N}} h(x) u_{\gamma, \lambda} d x+o(1) \\
= & \left(a+b \theta \alpha_{\gamma, \lambda}^{p(\theta-1)}\right)\left\|u_{\gamma, \lambda}\right\|^{p}-\gamma\left\|u_{\gamma, \lambda}\right\|_{H}^{p}-\lambda\left\|u_{\gamma, \lambda}\right\|_{q, w}^{q} \\
& -\left\|u_{\gamma, \lambda}\right\|_{p_{s}^{*}, K}^{p^{*}}-\int_{\mathbb{R}^{N}} h(x) u_{\gamma, \lambda} d x .
\end{aligned}
$$

Now (3.5) yields $\left\|u_{\gamma, \lambda}\right\|_{q, w}=\left\|v_{n}\right\|_{q, w}+o(1),\left\|u_{\gamma, \lambda}\right\| \leq \alpha_{\gamma, \lambda}=\left\|v_{n}\right\|+o(1)$ as well as $\left\|u_{\gamma, \lambda}\right\|_{H} \leq\left\|v_{n}\right\|_{H}$ and $\left\|u_{\gamma, \lambda}\right\|_{p_{s}^{*}, K} \leq\left\|v_{n}\right\|_{p_{s}^{*}, K}$ as $n \rightarrow \infty$. Multiplying the expression in (3.6) by $1 / \theta p$ and subtracting it below, by (3.5) and the fact that either $\theta=1$ or $\gamma \leq 0$, we find as $n \rightarrow \infty$

$$
\begin{aligned}
m_{\gamma, \lambda} \leq & J_{\gamma, \lambda}\left(u_{\gamma, \lambda}\right) \leq \frac{a+b \alpha_{\gamma, \lambda}^{p(\theta-1)}}{p}\left\|u_{\gamma, \lambda}\right\|^{p}-\frac{\gamma}{p}\left\|u_{\gamma, \lambda}\right\|_{H}^{p}-\frac{\lambda}{q}\left\|u_{\gamma, \lambda}\right\|_{q, w}^{q} \\
& -\frac{1}{p_{s}^{*}}\left\|u_{\gamma, \lambda}\right\|_{p_{s}^{*}, K}^{p_{s}^{*}}-\int_{\mathbb{R}^{N}} h(x) u_{\gamma, \lambda} d x \\
= & \frac{a}{p}\left(1-\frac{1}{\theta}\right)\left\|u_{\gamma, \lambda}\right\|^{p}-\frac{\gamma}{p}\left(1-\frac{1}{\theta}\right)\left\|u_{\gamma, \lambda}\right\|_{H}^{p}-\lambda\left(\frac{1}{q}-\frac{1}{\theta p}\right)\left\|u_{\gamma, \lambda}\right\|_{q, w}^{q} \\
& -\left(\frac{1}{p_{s}^{*}}-\frac{1}{\theta p}\right)\left\|u_{\gamma, \lambda}\right\|_{p_{s}^{*}, K}^{p_{s}^{*}}-\left(1-\frac{1}{\theta p}\right) \int_{\mathbb{R}^{N}} h(x) u_{\gamma, \lambda} d x \\
\leq & \frac{a}{p}\left(1-\frac{1}{\theta}\right)\left\|v_{n}\right\|^{p}-\frac{\gamma}{p}\left(1-\frac{1}{\theta}\right)\left\|v_{n}\right\|_{H}^{p}-\lambda\left(\frac{1}{q}-\frac{1}{\theta p}\right)\left\|v_{n}^{+}\right\|_{q, w}^{q} \\
& -\left(\frac{1}{p_{s}^{*}}-\frac{1}{\theta p}\right)\left\|v_{n}^{+}\right\|_{p_{s}^{*}, K}^{p_{s}^{*}}-\left(1-\frac{1}{\theta p}\right) \int_{\mathbb{R}^{N}} h(x) v_{n} d x+o(1) \\
= & J_{\gamma, \lambda}\left(v_{n}\right)-\frac{1}{\theta p}\left\langle J_{\gamma, \lambda}^{\prime}\left(v_{n}\right), v_{n}\right\rangle_{E^{\prime}, E}+o(1)=m_{\gamma, \lambda},
\end{aligned}
$$


since $v_{n} \rightarrow u_{\gamma, \lambda}$ in $L^{\nu}\left(\mathbb{R}^{N}\right)$ by Lemma 2.1 and so

$$
\lim _{n \rightarrow \infty} \int_{\mathbb{R}^{N}} h(x) v_{n} d x=\int_{\mathbb{R}^{N}} h(x) u_{\gamma, \lambda} d x
$$

being $h \in L^{\nu^{\prime}}\left(\mathbb{R}^{N}\right)$.

In conclusion, $u_{\gamma, \lambda}$ is a minimizer of $J_{\gamma, \lambda}$ in $\bar{B}_{\rho}$ and $J_{\gamma, \lambda}\left(u_{\gamma, \lambda}\right)=$ $m_{\gamma, \lambda}<0<\alpha \leq J_{\gamma, \lambda}(u)$ for all $u \in \partial \bar{B}_{\rho}$ by Lemma 3.1. Thus in turn $u_{\gamma, \lambda} \in B_{\rho}$, so that $J_{\gamma, \lambda}^{\prime}\left(u_{\gamma, \lambda}\right)=0$ and this implies that $u_{\gamma, \lambda}$ is a nontrivial nonnegative solution of (1.9), as stated.

It remains to show the asymptotic behavior (1.6). Fix $\gamma$. We recall that either $\theta=1$ and $\gamma<a H$, or $\theta>1$ and $\gamma \leq 0$. We know that $0<\left\|u_{\gamma, \lambda}\right\|<\rho=\rho\left(\gamma^{+}, \lambda\right)$, where $\rho\left(\gamma^{+}, \lambda\right)$ satisfies the identity

$$
a-\frac{\gamma^{+}}{H}=p^{\prime} \frac{C_{w}^{q}}{q^{\prime}} \lambda \rho\left(\gamma^{+}, \lambda\right)^{q-p}+p^{\prime} S_{K}\left(p_{s}^{*}-1\right) \rho\left(\gamma^{+}, \lambda\right)^{p_{s}^{*}-p}
$$

for all $\lambda>0$ by $(3.2)$ and the definition of $\rho\left(\gamma^{+}, \lambda\right)$. This implies at once that

$$
\lim _{\lambda \rightarrow \infty} \rho\left(\gamma^{+}, \lambda\right)=0
$$

since $p<q<p_{s}^{*}$. This shows (1.6) and completes the proof.

\section{Further results for general nonlocal operators}

In this section, we show that Theorems 1.1 and 1.3 continue to hold when $(-\Delta)_{p}^{s}$ in $(1.1),(1.8)$, and (1.9) is replaced by the nonlocal integrodifferential operator $\mathcal{L}_{K}$, defined, along any function $\varphi \in C_{0}^{\infty}\left(\mathbb{R}^{N}\right)$, by

$$
\mathcal{L}_{\mathcal{K}}(\varphi)=2 \lim _{\varepsilon \rightarrow 0^{+}} \int_{\mathbb{R}^{N} \backslash B_{\varepsilon}(x)}|\varphi(x)-\varphi(y)|^{p-2}[\varphi(x)-\varphi(y)] \mathcal{K}(x-y) d x d y,
$$

where the singular kernel $\mathcal{K}: \mathbb{R}^{N} \backslash\{0\} \rightarrow \mathbb{R}^{+}$satisfies the following properties:

$\left(\mathcal{K}_{1}\right) m \mathcal{K} \in L^{1}\left(\mathbb{R}^{N}\right)$, where $m(x)=\min \left\{1,|x|^{p}\right\}$;

$\left(\mathcal{K}_{2}\right)$ there exists a number $\mathcal{K}_{0}>0$ such that $\mathcal{K}(x) \geq \mathcal{K}_{0}|x|^{-(N+p s)}$ for all $x$ in $\mathbb{R}^{N} \backslash\{0\}$.

Obviously, the operator $\mathcal{L}_{\mathcal{K}}$ reduces to the fractional $p$-Laplacian $(-\Delta)_{p}^{s}$, when $K(x)=|x|^{-N-p s}$ and usually $\mathcal{K}_{0} \leq 1$.

Let us denote by $D_{\mathcal{K}}^{s, p}\left(\mathbb{R}^{N}\right)$ the completion of $C_{0}^{\infty}\left(\mathbb{R}^{N}\right)$ with respect to

$$
[u]_{s, p, \mathcal{K}}=\left(\iint_{\mathbb{R}^{2 N}}|u(x)-u(y)|^{p} \mathcal{K}(x-y) d x d y\right)^{1 / p}
$$


which is well-defined by $\left(\mathcal{K}_{1}\right)$. Clearly, the embedding $D_{\mathcal{K}}^{s, p}\left(\mathbb{R}^{N}\right) \hookrightarrow$ $D^{s, p}\left(\mathbb{R}^{N}\right)$ is continuous, being

$$
[u]_{s, p} \leq \mathcal{K}_{0}^{-1 / p}[u]_{s, p, \mathcal{K}} \quad \text { for all } u \in D_{\mathcal{K}}^{s, p}\left(\mathbb{R}^{N}\right),
$$

by $\left(\mathcal{K}_{2}\right)$. Similarly, $E_{\mathcal{K}}$ denotes the completion of $C_{0}^{\infty}\left(\mathbb{R}^{N}\right)$ with respect to the norm

$$
\|u\|_{\mathcal{K}}=\left([u]_{s, p, \mathcal{K}}^{p}+\|u\|_{p, V}^{p}\right)^{1 / p} .
$$

Then $E_{\mathcal{K}}=\left(E_{\mathcal{K}},\|\cdot\|_{\mathcal{K}}\right)$ is a separable uniformly convex Banach space, adapting the arguments of Proposition A.9 of [3].

It is clear that the embeddings $E_{\mathcal{K}} \hookrightarrow W^{s, p}\left(\mathbb{R}^{N}\right) \hookrightarrow L^{p_{s}^{*}}\left(\mathbb{R}^{N}\right)$ are continuous by the above remarks and Lemma 2.1. A similar argument as in Lemma 2.1 of $[\mathbf{1 0}]$, combined with some ideas taken from Appendix B of [4], shows that the embedding $E_{\mathcal{K}} \hookrightarrow L^{q}\left(\mathbb{R}^{N}, w\right)$ is compact.

A (weak) entire solution of

$$
\begin{aligned}
M\left(\|u\|_{\mathcal{K}}^{p}\right)\left[\mathcal{L}_{\mathcal{K}}(u)\right. & \left.+V(x)|u|^{p-2} u\right]-\gamma \frac{|u|^{p-2} u}{|x|^{p s}} \\
= & \lambda f(x, u)+g(x, u)+K(x)\left(u^{+}\right)^{p_{s}^{*}-1} \quad \text { in } \mathbb{R}^{N}
\end{aligned}
$$

is a function $u \in E_{\mathcal{K}}$ such that

$$
\begin{aligned}
M\left(\|u\|_{\mathcal{K}}^{p}\right)\langle u, \varphi\rangle_{\mathcal{K}}-\gamma\langle u, \varphi\rangle_{H}= & \lambda \int_{\mathbb{R}^{N}} f(x, u) \varphi d x \\
& +\int_{\mathbb{R}^{N}} g(x, u) \varphi d x+\left\langle u^{+}, \varphi\right\rangle_{p_{s}^{*}, K}, \\
\langle u, \varphi\rangle_{\mathcal{K}}= & \langle u, \varphi\rangle_{s, p, \mathcal{K}}+\langle u, \varphi\rangle_{p, V}, \\
\langle u, \varphi\rangle_{s, p, \mathcal{K}}= & \iint_{\mathbb{R}^{2 N}}|u(x)-u(y)|^{p-2}[u(x)-u(y)] \\
& \cdot[\varphi(x)-\varphi(y)] \mathcal{K}(x-y) d x d y .
\end{aligned}
$$

It is worth pointing out, as in [2], that it is not restrictive to assume $\mathcal{K}$ to be even, since the odd part of $\mathcal{K}$ does not give any contribution in the integral above. Indeed, write $\mathcal{K}=\mathcal{K}_{e}+\mathcal{K}_{o}$, where for all $x \in \mathbb{R}^{N} \backslash\{0\}$

$$
\mathcal{K}_{e}(x)=\frac{\mathcal{K}(x)+\mathcal{K}(-x)}{2} \text { and } \quad \mathcal{K}_{o}(x)=\frac{\mathcal{K}(x)-\mathcal{K}(-x)}{2} .
$$

Then, it is evident that $\langle u, \varphi\rangle_{s, p, \mathcal{K}}=\iint_{\mathbb{R}^{2 N}}|u(x)-u(y)|^{p-2}[u(x)-u(y)] \cdot[\varphi(x)-\varphi(y)] \mathcal{K}_{e}(x-y) d x d y$ for all $u$ and $\varphi \in E_{\mathcal{K}}$. 
Actually, the entire solutions of problem (4.1) correspond to the critical points of the energy functional $J_{\gamma, \lambda, \mathcal{K}}: E_{\mathcal{K}} \rightarrow \mathbb{R}$, for all $u \in E_{\mathcal{K}}$ defined by

$$
J_{\gamma, \lambda, \mathcal{K}}(u)=\frac{1}{p}\left[\mathscr{M}\left(\|u\|_{\mathcal{K}}^{p}\right)-\gamma\|u\|_{H}^{p}\right]-H_{\lambda}(u),
$$

where $u \mapsto H_{\lambda}(u)$ is given exactly as in Section 2. Lemmas 2.2 and 2.3 continue to hold for all $\gamma \in\left(-\infty, a \mathcal{K}_{0} H\right)$ and all $\lambda>0$ by $\left(\mathcal{K}_{1}\right)$, with obvious changes in their proof. Similarly, Lemma 2.4 can be proved in almost the same way as before, provided that $\gamma \in\left(-\infty, \kappa \mathcal{K}_{0} H\right)$ and $\lambda>0$, where $\kappa$ is given in (1.5) as usually. Thus we have proved

Theorem 4.1. Suppose that (4.1) is non-degenerate, i.e., that (1.3) holds, and that $(\mathcal{M}),(V),(\mathcal{V}),(\mathcal{F})$, and $(\mathcal{G})$ are satisfied. Then for every $\gamma \in\left(-\infty, \kappa \mathcal{K}_{0} H\right)$ problem (4.1) admits a nontrivial mountain pass solution $u_{\gamma, \lambda}$ for any $\lambda>0$, whenever $\|K\|_{\infty}=0$, and $u_{\gamma, \lambda}$ satisfies the asymptotic behavior (1.6). While, if $\|K\|_{\infty}>0$, then there exists a threshold $\lambda^{*}=\lambda^{*}(\gamma)>0$ such that for any $\lambda \geq \lambda^{*}$ problem (4.1) admits a nontrivial mountain pass solution $u_{\gamma, \lambda}$, satisfying again (1.6).

Moreover, if $g \equiv 0$, then the assertion above continues to hold assuming only condition $(V)$ on the potential $V$.

Finally, if $f, g: \mathbb{R}^{N} \times \mathbb{R} \rightarrow \mathbb{R}$ are Carathéodory functions, satisfying (1.7), and if $h$ is a nonnegative perturbation term of class $L^{\nu^{\prime}}\left(\mathbb{R}^{N}\right)$, where $\nu^{\prime}$ is the conjugate exponent of some fixed $\nu \in\left[p, p_{s}^{*}\right]$, then the non-homogeneous equation associated with (4.1), that is

$$
\begin{aligned}
M\left(\|u\|_{\mathcal{K}}^{p}\right) & {\left[\mathcal{L}_{\mathcal{K}}(u)+V(x)|u|^{p-2} u\right]-\gamma \frac{|u|^{p-2} u}{|x|^{p s}} } \\
& =\lambda f(x, u)+g(x, u)+K(x)\left(u^{+}\right)^{p_{s}^{*}-1}+h(x) \text { in } \mathbb{R}^{N},
\end{aligned}
$$

admits only nonnegative solutions in $\mathbb{R}^{N}$, provided that $\lambda \geq 0$ and $\gamma<a \mathcal{K}_{0} H$.

Clearly, also Theorem 1.2 continues to hold assuming in addition that the new singular kernel $\mathcal{K}$ is radial, that is

Theorem 4.2. Let $N \geq 2$ and let (1.3) hold. Assume that $(\mathcal{M}),(V)$, $(\mathcal{F})$, and $(\mathcal{G})$ are satisfied and that $K, \mathcal{K}, V, f$, and $g$ are radial functions in $x$. Then for every $\gamma \in(-\infty, \kappa H)$ problem (4.1) admits a nontrivial radial mountain pass solution $u_{\gamma, \lambda}$ for any $\lambda>0$, whenever $\|K\|_{\infty}=0$ and $u_{\gamma, \lambda}$ satisfies the asymptotic behavior (1.6). While if $\|K\|_{\infty}>0$, then there exists $\lambda^{*}=\lambda^{*}(\gamma)>0$ such that for any $\lambda \geq \lambda^{*}$ problem (4.1) admits a nontrivial radial mountain pass solution $u_{\gamma, \lambda}$, which satisfies again (1.6). 
The results of Section 3 continue to hold for the obvious generalization of (1.9) in $\mathbb{R}^{N}$, that is

$$
\begin{aligned}
M\left(\|u\|_{\mathcal{K}}^{p}\right)\left[\mathcal{L}_{\mathcal{K}}(u)+V(x)|u|^{p-2} u\right]-\gamma \frac{|u|^{p-2} u}{|x|^{p s}} \\
=\lambda w(x)\left(u^{+}\right)^{q-1}+K(x)\left(u^{+}\right)^{p_{s}^{*}-1}+h(x) .
\end{aligned}
$$

In particular, Lemma 3.1 is valid for all $\gamma \in\left(-\infty, a \mathcal{K}_{0} H\right)$ and all $\lambda \geq 0$. We have so proved

Theorem 4.3. Suppose that $(V)$ and (1.10) hold. Then for all $\gamma$ in $\left(-\infty, a \mathcal{K}_{0} H\right)$ and $\lambda>0$ there exists $\delta=\delta\left(\gamma^{+}, \lambda\right)>0$ such that for all nontrivial nonnegative perturbations $h \in L^{\nu^{\prime}}\left(\mathbb{R}^{N}\right)$, with $0<\|h\|_{\nu^{\prime}} \leq \delta$, problem (1.9) admits a nontrivial nonnegative solution $u_{\gamma, \lambda}$, provided that either $\theta=1$ in (1.10), or $\theta>1$ and $\gamma \leq 0$. Furthermore, the solution $u_{\gamma, \lambda}$ satisfies (1.6).

\section{Acknowledgements}

This paper has been started while P. Pucci was visiting the Department of Mathematics of the City University of Hong Kong and she thanks the department for the hospitality. P. Pucci was partly supported by the Italian MIUR project (201274FYK7) titled Variational and perturbative aspects of nonlinear differential problems and is a member of the Gruppo Nazionale per l'Analisi Matematica, la Probabilità e le loro Applicazioni (GNAMPA) of the Istituto Nazionale di Alta Matematica (INdAM). The manuscript was realized within the auspices of the INdAM-GNAMPA Project Problemi variazionali su varietà Riemanniane e gruppi di Carnot (Prot_2016_000421).

\section{References}

[1] A. Ambrosetti and P. H. Rabinowitz, Dual variational methods in critical point theory and applications, J. Functional Analysis 14(4) (1973), 349-381. DOI: 10.1016/0022-1236(73)90051-7.

[2] G. Autuori, A. Fiscella, And P. Pucci, Stationary Kirchhoff problems involving a fractional elliptic operator and a critical nonlinearity, Nonlinear Anal. 125 (2015), 699-714. DOI : 10.1016/j.na. 2015.06 .014$.

[3] G. Autuori And P. PuCci, Existence of entire solutions for a class of quasilinear elliptic equations, NoDEA Nonlinear Differential Equations Appl. 20(3) (2013), 977-1009. DOI : 10.1007/s00030-0120193-y. 
[4] G. Autuori And P. PuCci, Elliptic problems involving the fractional Laplacian in $\mathbb{R}^{N}$, J. Differential Equations 255(8) (2013), 2340-2362. DOI: 10.1016/j.jde.2013.06.016.

[5] B. Barrios, M. Medina, And I. Peral, Some remarks on the solvability of non-local elliptic problems with the Hardy potential, Commun. Contemp. Math. 16(4) (2014), 1350046, 29 pp. DOI: 10.1142/S0219199713500466.

[6] T. Bartsch And Z. Q. WANG, Existence and multiplicity results for some superlinear elliptic problems on $\mathbf{R}^{N}$, Comm. Partial Differential Equations 20(9-10) (1995), 1725-1741. DOI: 10.1080/ 03605309508821149.

[7] H. BrÉzis AND E. LiEB, A relation between pointwise convergence of functions and convergence of functionals, Proc. Amer. Math. Soc. 88(3) (1983), 486-490. DOI : $10.2307 / 2044999$.

[8] L. A. CAfFarelli, Some nonlinear problems involving non-local diffusions, in: "ICIAM 0\%-6th International Congress on Industrial and Applied Mathematics", Eur. Math. Soc., Zürich, 2009, pp. 43-56. DOI: 10.4171/056-1/3.

[9] L. A. CAfFARELli, Non-local diffusions, drifts and games, in: "Nonlinear Partial Differential Equations", Abel Symp. 7, Springer, Heidelberg, 2012, pp. 37-52. DOI : 10.1007/978-3-642-25361-4_3.

[10] M. CAponi And P. PuCCI, Existence theorems for entire solutions of stationary Kirchhoff fractional $p$-Laplacian equations, Ann. Mat. Pura Appl. (4) 195(6) (2016), 2099-2129. DOI: 10.1007/s10231016-0555-x.

[11] G. Cerami, X. Zhong, and W. Zou, On some nonlinear elliptic PDEs with Sobolev-Hardy critical exponents and a $\mathrm{Li}-\mathrm{Lin}$ open problem, Calc. Var. Partial Differential Equations 54(2) (2015), 1793-1829. DOI : 10.1007/s00526-015-0844-z.

[12] X. Chang, Ground states of some fractional Schrödinger equations on $\mathbb{R}^{N}$, Proc. Edinb. Math. Soc. (2) 58(2) (2015), 305-321. DOI: 10.1017/S0013091514000200.

[13] S. Chen And S. LiU, Standing waves for 4-superlinear SchrödingerKirchhoff equations, Math. Methods Appl. Sci. 38(11) (2015), 2185-2193. DOI : 10.1002/mma.3212.

[14] D. C. De Morais Filho, M. A. S. Souto, And J. M. Do O., A compactness embedding lemma, a principle of symmetric criticality and applications to elliptic problems, Proyecciones 19(1) (2000), 1-17. DOI: $10.4067 /$ S0716-09172000000100001. 
[15] E. Di Nezza, G. Palatucci, And E. Valdinoci, Hitchhiker's guide to the fractional Sobolev spaces, Bull. Sci. Math. 136(5) (2012), 521-573. DOI: 10.1016/j.bulsci.2011.12.004.

[16] H. FAN AND X. LiU, Positive and negative solutions for a class of Kirchhoff type problems on unbounded domain, Nonlinear Anal. 114 (2015), 186-196. DOI: 10.1016/j.na.2014.07.012.

[17] P. FElmer And C. Torres, Radial symmetry of ground states for a regional fractional nonlinear Schrödinger equation, Commun. Pure Appl. Anal. 13(6) (2014), 2395-2406. DOI: 10.3934/cpaa. 2014.13.2395.

[18] P. Felmer And I. Vergara, Scalar field equation with non-local diffusion, NoDEA Nonlinear Differential Equations Appl. 22(5) (2015), 1411-1428. DOI : 10.1007/s00030-015-0328-z.

[19] G. M. Figueiredo, Existence of a positive solution for a Kirchhoff problem type with critical growth via truncation argument, J. Math. Anal. Appl. 401(2) (2013), 706-713. DOI: 10.1016/j.jmaa.2012. 12.053.

[20] G. M. Figueiredo And J. R. Santos Júnior, Existence of a least energy nodal solution for a Schrödinger-Kirchhoff equation with potential vanishing at infinity, J. Math. Phys. 56(5) (2015), 051506, 18 pp. DOI : 10.1063/1.4921639.

[21] A. Fiscella AND P. PuCCI, On certain nonlocal Hardy-Sobolev critical elliptic Dirichlet problems, Adv. Differential Equations 21(5-6) (2016), 571-599.

[22] A. Fiscella And E. VAldinoci, A critical Kirchhoff type problem involving a nonlocal operator, Nonlinear Anal. 94 (2014), 156-170. DOI: $10.1016 / j . n a .2013 .08 .011$.

[23] R. L. Frank And R. SEIRINGER, Non-linear ground state representations and sharp Hardy inequalities, J. Funct. Anal. 255(12) (2008), 3407-3430. DOI : 10.1016/j.jfa.2008.05.015.

[24] B. GE And C. Zhang, Existence of a positive solution to Kirchhoff problems involving the fractional Laplacian, Z. Anal. Anwend. 34(4) (2015), 419-434. DOI: 10.4171/ZAA/1547.

[25] Y. GUO AND J. NiE, Existence and multiplicity of nontrivial solutions for $p$-Laplacian Schrödinger-Kirchhoff-type equations, $J$. Math. Anal. Appl. 428(2) (2015), 1054-1069. DOI: 10.1016/j.jmaa. 2015.03 .064 .

[26] A. LI AND J. Su, Existence and multiplicity of solutions for Kirchhoff-type equation with radial potentials in $\mathbb{R}^{3}, Z$. Angew. Math. Phys. 66(6) (2015), 3147-3158. DOI: 10.1007/s00033-0150551-9. 
[27] L. LI AND J.-J. Sun, Existence and multiplicity of solutions for the Kirchhoff equations with asymptotically linear nonlinearities, Nonlinear Anal. Real World Appl. 26 (2015), 391-399. DOI: 10.1016/j . nonrwa.2015.07.002.

[28] P.-L. Lions, Symétrie et compacité dans les espaces de Sobolev, J. Funct. Anal. 49(3) (1982), 315-334. DOI : 10.1016/0022-1236(82) 90072-6.

[29] L. LiU And C. Chen, Study on existence of solutions for $p$-Kirchhoff elliptic equation in $\mathbb{R}^{N}$ with vanishing potential, J. Dyn. Control Syst. 20(4) (2014), 575-592. DOI: 10.1007/s10883-0149244-5.

[30] J. Liu, J.-F. LiaO, And C. L. TAng, Positive solutions for Kirchhoff-type equations with critical exponent in $\mathbb{R}^{N}$, J. Math. Anal. Appl. 429(2) (2015), 1153-1172. DOI: 10.1016/j.jmaa. 2015. 04.066.

[31] V. Maz'ya And T. Shaposhnikova, On the Bourgain, Brezis, and Mironescu theorem concerning limiting embeddings of fractional Sobolev spaces, J. Funct. Anal. 195(2) (2002), 230-238. DOI: 10.1006/jfan. 2002. 3955 .

[32] X. Mingqi, G. Molica Bisci, G. Tian, and B. Zhang, Infinitely many solutions for the stationary Kirchhoff problems involving the fractional p-Laplacian, Nonlinearity 29(2) (2016), 357-374. DOI : $10.1088 / 0951-7715 / 29 / 2 / 357$.

[33] N. NyAmORADI, Existence of three solutions for Kirchhoff nonlocal operators of elliptic type, Math. Commun. 18(2) (2013), 489-502.

[34] R. S. Palais, The principle of symmetric criticality, Comm. Math. Phys. 69(1) (1979), 19-30.

[35] P. PuCCI AND S. SAldi, Critical stationary Kirchhoff equations in $\mathbb{R}^{N}$ involving nonlocal operators, Rev. Mat. Iberoam. 32(1) (2016), 1-22. DOI: 10.4171/RMI/879.

[36] P. Pucci, M. Xiang, And B. Zhang, Multiple solutions for nonhomogeneous Schrödinger-Kirchhoff type equations involving the fractional $p$-Laplacian in $\mathbb{R}^{N}$, Calc. Var. Partial Differential Equations 54(3) (2015), 2785-2806. DOI: 10.1007/s00526-015-0883-5.

[37] P. Pucci, M. Xiang, And B. Zhang, Existence and multiplicity of entire solutions for fractional $p$-Kirchhoff equations, Adv. Nonlinear Anal. 5(1) (2016), 27-55. DOI: 10.1515/anona-2015-0102.

[38] P. PuCCI AND Q. Zhang, Existence of entire solutions for a class of variable exponent elliptic equations, J. Differential Equations 257(5) (2014), 1529-1566. DOI: 10.1016/j.jde.2014.05.023. 
[39] X. Ros-Oton, Nonlocal elliptic equations in bounded domains: a survey, Publ. Mat. 60(1) (2016), 3-26. DOI: 10.5565/PUBLMAT_ 60116_01.

[40] S. SECCHI, Ground state solutions for nonlinear fractional Schrödinger equations in $\mathbb{R}^{N}$, J. Math. Phys. 54(3) (2013), 031501, 17 pp. DOI: $10.1063 / 1.4793990$.

[41] C. E. Torres Ledesma, Multiplicity result for non-homogeneous fractional Schrödinger-Kirchhoff type equations in $\mathbb{R}^{n}$, Adv. Nonlinear Anal. (2016), 31 pp. DOI: 10.1515/anona-2015-0096.

[42] J. L. VÁzQUEZ, Nonlinear diffusion with fractional Laplacian operators, in: "Nonlinear Partial Differential Equations", Abel Symp. 7, Springer, Heidelberg, 2012, pp. 271-298. DOI : 10.1007/978-3-64225361-4_ 15.

[43] J. L. VÁzquez, Recent progress in the theory of nonlinear diffusion with fractional Laplacian operators, Discrete Contin. Dyn. Syst. Ser. $S$ 7(4) (2014), 857-885. DOI: 10.3934/dcdss.2014.7.857.

[44] Q. XIE AND S. MA, Existence and concentration of positive solutions for Kirchhoff-type problems with a steep well potential, $J$. Math. Anal. Appl. 431(2) (2015), 1210-1223. DOI: 10.1016/j.jmaa. 2015.05 .027$.

[45] Y. YE AND C.-L. TANG, Multiple solutions for Kirchhoff-type equations in $\mathbb{R}^{N}$, J. Math. Phys. 54(8) (2013), 081508, 16 pp. DOI: $10.1063 / 1.4819249$

Paolo Piersanti:

Department of Mathematics

City University of Hong Kong

Tat Chee Avenue, Kowloon

Hong Kong

E-mail address: ppiersan-c@my.cityu.edu.hk

Patrizia Pucci:

Dipartimento di Matematica e Informatica

Università degli Studi di Perugia

Via Vanvitelli 1

06123 Perugia

Italy

E-mail address: patrizia.pucci@unipg.it

Primera versió rebuda el 14 de març de 2016, darrera versió rebuda el 30 de juny de 2016 . 